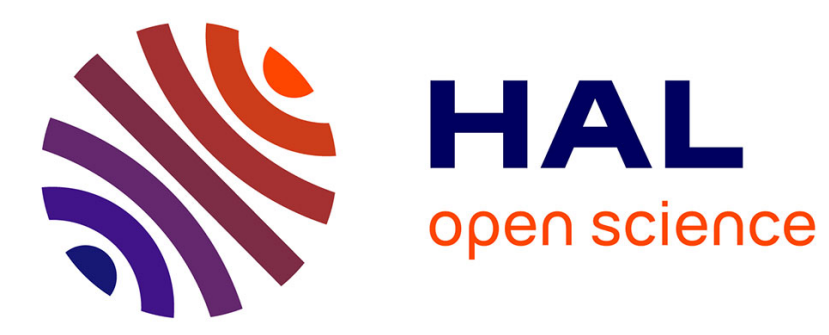

\title{
Aircraft deconfliction via Mathematical Programming: Review and insights
}

Mercedes Pelegrín, Claudia d'Ambrosio

\section{To cite this version:}

Mercedes Pelegrín, Claudia d'Ambrosio. Aircraft deconfliction via Mathematical Programming: Review and insights. 2020. hal-02902566v2

\section{HAL Id: hal-02902566 \\ https://hal.science/hal-02902566v2}

Preprint submitted on 21 Dec 2020 (v2), last revised 8 Mar 2021 (v3)

HAL is a multi-disciplinary open access archive for the deposit and dissemination of scientific research documents, whether they are published or not. The documents may come from teaching and research institutions in France or abroad, or from public or private research centers.
L'archive ouverte pluridisciplinaire HAL, est destinée au dépôt et à la diffusion de documents scientifiques de niveau recherche, publiés ou non, émanant des établissements d'enseignement et de recherche français ou étrangers, des laboratoires publics ou privés. 


\title{
Aircraft deconfliction via Mathematical Programming: Review and insights
}

\author{
Mercedes Pelegrín ${ }^{1}$, Claudia D'Ambrosio ${ }^{1}$
}

\begin{abstract}
Computer-aided Air Traffic Management has increasingly attracted the interest of the Operations Research community. This includes, among other tasks, the design of decision support tools for detection and resolution of conflict situations on flight. Even if numerous optimization approaches have been proposed, there has been little debate towards homogenization. We synthesize the efforts made by the Operations Research community in the past few decades to provide mathematical models to aid conflict detection and resolution at tactical level. Different mathematical representations of aircraft separation conditions are presented in a unifying analysis. The models, which hinge on these conditions, are then revisited, providing insight into their computational performance.
\end{abstract}

Keywords: air traffic control; optimization; conflict detection and resolution; separation conditions

\section{Introduction}

Air traffic control (ATC) is a challenging field where complex decisions have to be made in short time. The increase of daily volume of flights engenders the need of decision making tools to support manual control in order to improve airspace capacity while ensuring safety. The urgency for automation or support tools within ATC systems has been observed in a number of corporate initiatives including the NextGen - Next Generation Air Transportation System - project in the US (see Hansman 2012) and the SESAR - Single European Sky Air Traffic Management Research-

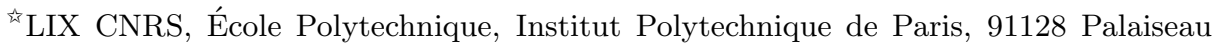
France

Email addresses: pelegringarcia@lix.polytechnique.fr (Mercedes Pelegrín), dambrosio@lix.polytechnique.fr (Claudia D'Ambrosio)
} 
(see European Commission \& EUROCONTROL 2009) and ERASMUSEn Route Air traffic Soft Management Ultimate System- (see Brochard 2005) projects in Europe. On the other hand, flying vehicles daily volume is expected to grow in the near future, especially due to the development of technology and its emerging application in urban air mobility. As a result, there is a growing interest in decision-making approaches for ATC, which is being captured by the Operations Research (OR) community.

Congested airspace can lead to loss of separation between aircraft, which has to be avoided to guarantee safety. Ensuring aircraft separation during flight is one of the major tasks of ATC and is known as conflict detection and resolution (CDR) or aircraft deconfliction. CDR takes place at different stages. First, it is performed on the earliest planning of the flights. This is known as strategic deconfliction and usually occurs before departure; however, it is not limited to a priori actions, in particular when the duration of flights is long, see Rios (2018), Liang et al. (2014). The following stage involves tactical deconfliction, which usually takes place during flight, generally from five to thirty minutes before conflicts occur. Finally, collision avoidance stage is the latest avoidance of hazard, aimed at addressing imminent conflicts (in less than one minute). Congestion also affects other segments of ATC, such as airport traffic, which is currently one of the bottlenecks of ATM systems, see for example Wu \& Caves (2002), Fairbrother et al. (2020), Samà et al. (2017).

Tactical CDR has been typically modelled as an optimization problem in which aircraft trajectories are corrected to ensure that a minimum separation is maintained. At tactical level, specific operational aspects have to be considered, which principally affect the changes allowed for trajectories. Feasible maneuvers are typically classified into three types, namely: speed changes (acceleration or deceleration), heading angle changes (right or left bearing), and vertical changes (flight level reallocation). All of these maneuvers are limited by some operational bounds. The goal of optimization varies between works and includes maximizing the number of solved conflicts, having equally affected flights (fairness), minimizing deviation from nominal aircraft trajectories and economic factors such as fuel consumption or total delay.

According to the International Civil Aviation Organization (see ICAO 1996), aircraft must be separated by at least $5 \mathrm{NM}$ horizontally and $1000 \mathrm{ft}$ vertically during the flight, which yields a safety cylinder as shown in Figure 1. A pair of aircraft violating at least one of these rules are said to be in conflict; one can speak respectively of horizontal or vertical conflicts. At the cruising phase, airspace is segregated in independent flight levels, see Mori 


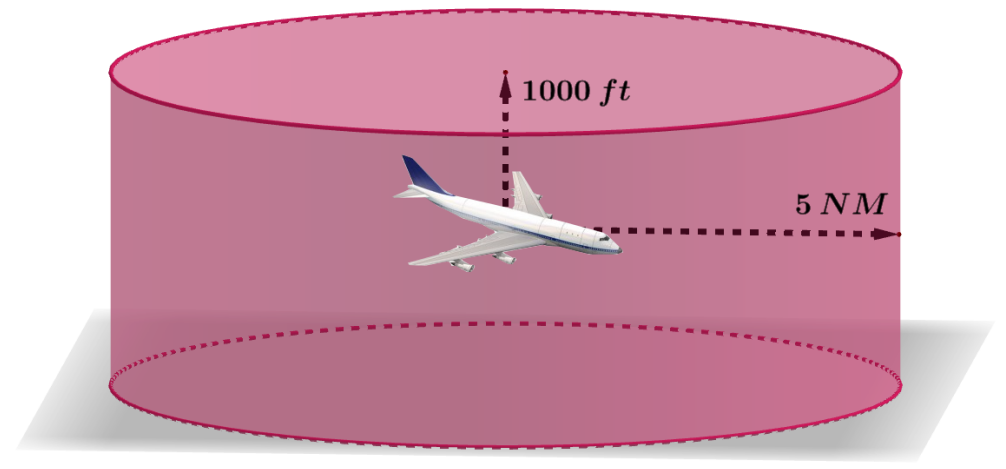

Figure 1: Safety cylinder around aircraft

(2017). Therefore, tactical deconfliction usually assumes that all aircraft fly at the same flight level, eliminating the need to address vertical conflicts. Once the problem dimension is fixed (2D for horizontal conflicts and 3D in the more general case), aircraft trajectories can be modelled by different kinds of equations. In the most simple case, they are linear functions of time, that is, aircraft motion is supposed to be rectilinear. Alternatively, if a constant acceleration is assumed, aircraft position is a quadratic function of time. Another common approach is to consider piece-wise linear functions to represent trajectories. Regarding the modelling of aircraft maneuvers, there also exists a range of possible choices. The most frequent assumption among the approaches covered here is that all aircraft will execute maneuvers at the beginning of the time horizon and instantaneously. On the contrary, more complex models allow aircraft to change their trajectories at different time instants and consider aircraft kinetics during maneuvers. Trajectory recovery after conflict resolution is sometimes considered as well. The right choice of modelling assumptions depends on the specific problem requirements and varies between applications. Also, it is important to look for a trade-off between flexibility and tractability of the resulting optimization problem.

Leaving aside technical modelling aspects and moving to an OR perspective, CDR involves two types of decisions. The first and most evident is the modification of aircraft trajectories, which will be expressed in terms of speed, heading or altitude changes, or a combination of them. Possible variables such as the new kilometres or miles per hour or the bearing angle take (in principle) their values in continuous domains. Therefore, they are naturally modelled with continuous decision variables. The second type of 
decision is not obvious at first sight: one needs to investigate the problem further to identify it. This will be extensively addressed in next sections, but for the moment we just say that there are some combinatorial decisions characterizing CDR and standing for the choice among possible scenarios. Such combinatorial decisions are typically modelled in OR models with binary variables. Therefore, a natural mathematical programming formulation for CDR will be a Mixed Integer Linear Program (MILP) or a Mixed Integer Nonlinear Program (MINLP) - constraints and objective function will be linear or nonlinear depending on the modelling choices made.

Although CDR optimization was originally proposed in the context of ATC, it is nowadays gaining importance in other arising domains. For instance, urban shared mobility become popular in recent years (see Mourad et al. 2019) and will soon be part of our skies, yielding a new layer of air traffic. In addition, collision avoidance is a hot topic in the field of autonomous vehicles, including unmanned aerial vehicles (UAVs), see, for example, Huang et al. (2019). This interest is motivated by the potential applications of these vehicles in commercial, military, or governmental contexts. Finally, algorithms and protocols for collision-free trajectories are essential in the design of control architectures of robots, see Bareiss \& den Berg (2013). This includes, among others, material handling vehicles in warehouses or production plants, which transport raw materials or perform tasks in production processes.

This work aims at providing a unified mathematical framework for tactical CDR. Special emphasis is placed on the mathematical representation of aircraft separation conditions. This has been represented through different equations, which we revisit here. Existing alternative separation equations are motivated, explained, and compared, showing the relation between them. Mathematical Programming models for CDR are then revised, presenting a total of 10 mathematical formulations under a unified notation. Even if there exist other reviews on closely related topics (Barnhart et al. (2003) presents a review on OR applications in air transport including scheduling, routing, crew assignment and ATC, among other topics; Kuchar \& Yang (2000) gives an overview on CDR methods), to the best of our knowledge this is the first work synthesizing Mathematical Programming approaches for tactical CDR.

The rest of the paper is organized as follows. In Section 2, we formally state the problem and fix some notation. Section 3 is devoted to present and analyze the different mathematical representations of separation constraints. An explanatory exposition of the different formulas and their interrelation is also provided. Since separation constraints are the core of CDR, this 
analysis is essential for Section 4, where some mathematical programming formulations are presented. Section 5 gives details about the performance of the state-of-the-art approaches. Finally, Section 6 discusses current challenges in CDR and future research directions, and Section 7 closes the paper with some conclusions.

\section{Problem definition}

In ATC for aircraft deconfliction, a particular sector of the airspace is tracked during a given time horizon, $[0, T]$. During this time window, a set of aircraft, $\mathcal{A}$, traverses the observed sector. We consider control actions at a tactical level, that is, they are taken some minutes before potential conflicts occur (values of $T$ usually range between 10 and 20 minutes). Aircraft nominal trajectories can be described through a family of functions, $\hat{p}_{i}(t):[0, T] \rightarrow \mathbb{R}^{3}$, which give the position of aircraft $i \in \mathcal{A}$ at instant $t$ of the time horizon according to a nominal plan. If we denote by $d$ the minimum separation distance required between aircraft, the CDR problem can be stated as follows: " find new trajectories $p_{i}(t):[0, T] \rightarrow \mathbb{R}^{3}$ satisfying operational restrictions and such that, for all pair of aircraft $i, j \in \mathcal{A}$ and time instant $t \in[0, T],\left\|p_{i}(t)-p_{j}(t)\right\| \geq d$ ", where $\|\cdot\|$ denotes the Euclidean norm.

As mentioned in the introduction, aircraft are usually assumed to have either a rectilinear (null acceleration) or uniformly accelerated (constant acceleration) motion. Most of the works covered in this review are of the first type, but we also discuss examples of the second type such as Omer \& Farges (2013), and Omer (2015). In our analysis, in order to fix ideas and given that this is the case of most of the approaches, we assume $p_{i}(t)$ to be linear functions. Namely,

$$
p_{i}(t)=\hat{p}_{i}+V_{i} t
$$

where $\hat{p}_{i}:=\hat{p}_{i}(0)$ is the initial position of aircraft $i$ and $V_{i}$ is its vector of modified velocity. These equations of motion are the base upon which most of the mathematical constraints for aircraft separation are built, as we discuss in next section. This is the main reason for setting $p_{i}(t)$ as above, although in general the reader can think on the CDR without restricting aircraft position equations. Position vectors are very often assumed to be in $\mathbb{R}^{2}$, which corresponds to aircraft flying at the same flight level. Therefore, we denote initial position and vector of velocity components by $\hat{p}_{i}=\left(\hat{x}_{i}, \hat{y}_{i}\right)$ and $V_{i}=\left(V_{x i}, V_{y i}\right)$, respectively. Similarly to new trajectories, nominal ones are represented by $\hat{p}_{i}(t)=\hat{p}_{i}+\hat{V}_{i} t$, where $\hat{V}_{i}$ denotes aircraft nominal velocity. 
Operational limitations typically result in mathematical constraints on the vectors of aircraft velocities. Let us denote by $v_{i}$ the (scalar) speed of $i$ and by $\theta_{i}$ its heading angle. These variables are subject to lower and upper bounds $\underline{v}_{i}, \bar{v}_{i}$ and $\underline{\theta}_{i}, \bar{\theta}_{i}$, respectively. Note that we save the term velocity to refer a vector of velocity, which comprises information on the heading angle and speed of the aircraft. The term speed is thus saved for a scalar magnitude and corresponds to the Euclidean norm of the vector of velocity. This way, speed $v_{i}$ and heading angle $\theta_{i}$ characterize the $i$-th velocity vector as follows

$$
V_{i}=\left(v_{i} \cos \theta_{i}, v_{i} \sin \theta_{i}\right) .
$$

However, the new speed and heading angle of an aircraft can be also represented by using decision variables corresponding to the relative modification made to the nominal values. Typically,

$$
v_{i}=q_{i} \hat{v}_{i} \quad \text { and } \quad \theta_{i}=\hat{\theta}_{i}+\omega_{i}
$$

where $\hat{v}_{i}$ and $\hat{\theta}_{i}$ are the nominal speed and heading angle of $i, q_{i} \geq 0$ is a scale factor ( $q_{i}=1$ means no speed change) and $\omega_{i}$ is the variation of the heading angle $i$ ( $\omega_{i}=0$ means no bearing). Scale factor $q_{i}$ is usually considered to range between 0.94 and 1.03, which means modifying the speed between a $-6 \%$ and $3 \%$ of its nominal value. According to the European aeronautical project ERASMUS (see Brochard 2005), this allows a subliminal control, yielding modifications that are barely perceived by air traffic controllers.

Different versions of the CDR problem, which differ on the type of maneuvers allowed, have been studied in the literature. The most prominent ones, are those in which only speed changes (SC) are allowed, only heading angle changes (HAC) are permitted and that in which both changes are contemplated (SHAC). Since airspace is usually assumed to be divided into flight levels, there is not a specific variant for altitude changes. One of the previous configurations, namely SC, HAC, or SHAC, is supposed for each flight level instead. The following definition uses the notation above to give a mathematical statement of the CDR in its more general version.

Definition 1 (SHAC). Given a set of aircraft $\mathcal{A}$ and time horizon $[0, T]$, suppose that each $i \in \mathcal{A}$ has initial position $\hat{p}_{i}$ and nominal speed and heading angle $\hat{v}_{i}$ and $\hat{\theta}_{i}$, respectively. The conflict detection and resolution problem is to find new speeds and heading angles $v_{i}$ and $\theta_{i}$ such that

(i) $\left\|p_{i}(t)-p_{j}(t)\right\|=\left\|\hat{p}_{i}+V_{i} t-\left(\hat{p}_{j}+V_{j} t\right)\right\| \geq d$ for all $i, j \in \mathcal{A}$ and $t \in[0, T]$, where $V_{i}=\left(v_{i} \cos \theta_{i}, v_{i} \sin \theta_{i}\right)$ for all $i \in \mathcal{A}$; 
(ii) given the control bounds $\underline{q}_{i}, \bar{q}_{i}$ and $\underline{\omega}_{i}, \bar{\omega}_{i}$ for every $i \in \mathcal{A}$, there is $q_{i}$ and $\omega_{i}$ such that

$$
\begin{aligned}
& \text { - } v_{i}=q_{i} \hat{v}_{i}, \quad \theta_{i}=\hat{\theta}_{i}+\omega_{i}, \\
& \text { - } \underline{q}_{i} \leq q_{i} \leq \bar{q}_{i}, \quad \underline{\omega}_{i} \leq \omega_{i} \leq \bar{\omega}_{i} .
\end{aligned}
$$

In addition, a certain function of the changes made should be minimized.

Condition (i) in the previous definition is the core of the CDR problem, and it is known as the separation condition. Two initial observations are that it is nonlinear because of the Euclidean norm and that it depends on time. Section 3 is entirely devoted to discuss the separation condition and how it has been modelled in the literature using different approaches. Before that, we summarize some useful notation.

\subsection{Notation}

In addition to the already introduced terminology, there are other elements characterizing the CDR problem that appear in the literature in a recurring way. For instance, relative positions and velocity vectors of pairs of aircraft are key for mathematical representation of separation, as well as some relative angles. We have unified existing mathematical notation to present the different approaches in the literature within a common working framework. The mathematical notation of this paper can be summarized as follows:

- Problem input

$-\mathcal{A} \quad$ set of aircraft, $i, j \in \mathcal{A}$

$-d \quad$ safety distance between aircraft, in $N M$

- $\hat{p}_{i}=\left(\hat{x}_{i}, \hat{y}_{i}\right) \quad$ initial position of aircraft $i \in \mathcal{A}$

- $\hat{v}_{i} \quad$ magnitude of nominal speed of aircraft $i \in \mathcal{A}$, in $N M / h$

- $\hat{\theta}_{i} \quad$ nominal heading angle of aircraft $i \in \mathcal{A}$, in radians

- $\hat{V}_{i}=\left(\hat{v}_{i} \cos \hat{\theta}_{i}, \hat{v}_{i} \sin \hat{\theta}_{i}\right) \quad$ nominal vector of velocity of $i \in \mathcal{A}$

- $\hat{p}_{i j}:=\hat{p}_{i}-\hat{p}_{j}$ vector difference, $\hat{p}_{i j}=\left(\hat{x}_{i j}, \hat{y}_{i j}\right)$, with $\hat{x}_{i j}:=\hat{x}_{i}-$ $\hat{x}_{j}, \hat{y}_{i j}:=\hat{y}_{i}-\hat{y}_{j}, i, j \in \mathcal{A}$

- $\hat{d}_{i j} \quad$ initial distance between aircraft $i$ and $j$ (coincides with $\left.\left\|\hat{p}_{i j}\right\|\right), i, j \in \mathcal{A}$

- $\beta_{i j} \quad$ slope of the line joining $i$ and $j$ (coincides with heading angle of $\left.\hat{p}_{i j}\right), i, j \in \mathcal{A}$ 
$-\alpha_{i j}:=\arcsin \left(\frac{d}{\hat{d}_{i j}}\right), i, j \in \mathcal{A}$

- Variables

- $v_{i} \quad$ magnitude of modified speed of aircraft $i \in \mathcal{A}$

- $\theta_{i} \quad$ modified heading angle of aircraft $i \in \mathcal{A}$

- $q_{i} \quad$ scale factor applied to nominal speed of aircraft $i \in \mathcal{A}, v_{i}=$ $q_{i} \hat{v}_{i}$

- $\omega_{i} \quad$ bearing applied to nominal heading angle of aircraft $i \in \mathcal{A}$, $\theta_{i}=\hat{\theta}_{i}+\omega_{i}$

- $V_{i}=\left(V_{x i}, V_{y i}\right) \quad$ modified vector of velocity of $i \in \mathcal{A}$. It can be expressed in different forms:

$$
\begin{gathered}
V_{i}=\left(v_{i} \cos \theta_{i}, v_{i} \sin \theta_{i}\right) \\
V_{i}=\left(q_{i} \hat{v}_{i} \cos \left(\hat{\theta}_{i}+\omega_{i}\right), q_{i} \hat{v}_{i} \sin \left(\hat{\theta}_{i}+\omega_{i}\right)\right)
\end{gathered}
$$

- $p_{i}(t):=\hat{p}_{i}+t V_{i}$ position of aircraft $i \in \mathcal{A}$ at instant $t \in[0, T]$

- $f_{i j}(t):=\left\|p_{i}(t)-p_{j}(t)\right\|^{2}$ squared distance between $i$ and $j$ at instant $t \in[0, T], i, j \in \mathcal{A}$

$-\Gamma\left(v_{i}, v_{j}, \gamma_{i j}\right)$ minimum difference between the instants in which $i$ and $j$ traverse the crossing point of their trajectories, which intersect with angle $\gamma_{i j}$.

$-V_{i j}:=V_{i}-V_{j} \quad$ modified relative velocity. We will denote its coordinates by $\left(V_{x i j}, V_{y i j}\right)$. Alternatively, it can be expressed as follows:

$$
\begin{gathered}
V_{i j}=\left(v_{i} \cos \theta_{i}-v_{j} \cos \theta_{j}, v_{i} \sin \theta_{i}-v_{j} \sin \theta_{j}\right) \text { or } \\
V_{i j}=\left(\left\|V_{i j}\right\| \cos \phi_{i j},\left\|V_{i j}\right\| \sin \phi_{i j}\right),
\end{gathered}
$$

where $\phi_{i j}$ is the heading angle of $V_{i j}$ (note $\phi_{i j} \neq \theta_{i}-\theta_{j}$ in general).

- Bounds

$-\underline{q}_{i}, \bar{q}_{i} \geq 0: \quad \underline{q}_{i} \leq q_{i} \leq \bar{q}_{i}$

- $\underline{\omega}_{i}, \bar{\omega}_{i}$ such that $\left[\underline{\omega}_{i}, \bar{\omega}_{i}\right] \subseteq[-\pi / 2, \pi / 2]: \quad \underline{\omega}_{i} \leq \omega_{i} \leq \bar{\omega}_{i}$

- $\underline{v}_{i}, \bar{v}_{i}$ such that $\underline{v}_{i}:=\underline{q}_{i} \hat{v}_{i}, \bar{v}_{i}:=\bar{q}_{i} \hat{v}_{i}: \quad \underline{v}_{i} \leq v_{i} \leq \bar{v}_{i}$

$-\underline{\theta}_{i}, \bar{\theta}_{i}$ such that $\underline{\theta}_{i}:=\hat{\theta}_{i}+\underline{\omega}_{i}, \bar{\theta}_{i}:=\hat{\theta}_{i}+\bar{\omega}_{i}: \quad \underline{\theta}_{i} \leq \theta_{i} \leq \bar{\theta}_{i}$ 


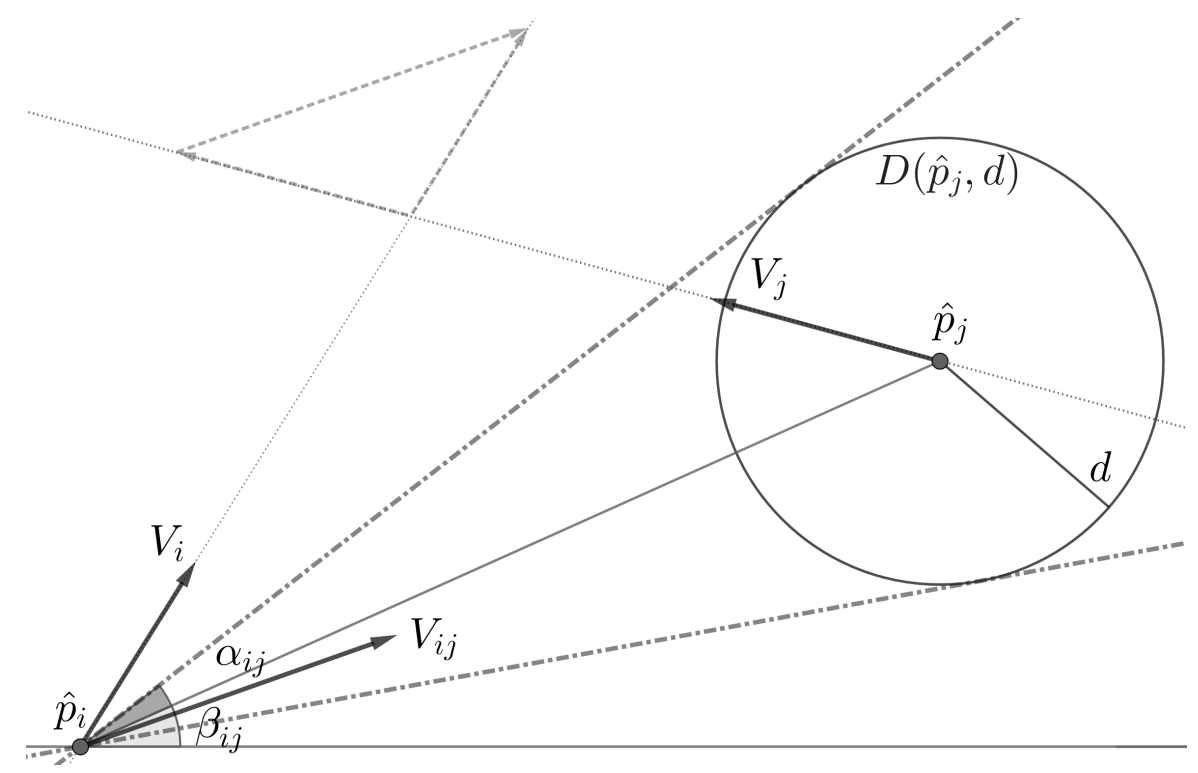

Figure 2: Geometrical analysis of conflict between two aircraft based on relative velocity

\section{The separation condition}

This section is devoted to the study of the so-called separation condition, namely

$$
\left\|p_{i}(t)-p_{j}(t)\right\| \geq d \quad \forall t \in[0, T], \forall i, j \in \mathcal{A}: i<j .
$$

Such condition cannot be directly plugged-in a mathematical programming solver, mainly because of the fact that one of its quantifiers is $t$, which ranges on a continuous domain. A natural way of dealing with this is to consider discrete time steps within $[0, T]$ at which (1) is then imposed, see Richards \& How (2002). If the lag between steps is sufficiently small, safety is ensured. However, time discretization is not the only valid approach here. Many authors used different versions of (1) that are not time dependent to write their models. In the following, we revise the different ways of translating (1) into mathematical programming constraints that have been proposed in the literature. For these separation constraints that are not indexed by a temporal component, $T=+\infty$ is implicitly assumed. For the sake of equations readability, we drop the condition $i<j$ in our analysis.

\subsection{Geometrical conditions}

We start with the simple observation that

$$
\left\|p_{i}(t)-p_{j}(t)\right\|=\left\|\hat{p}_{i}+t V_{i}-\left(\hat{p}_{j}+t V_{j}\right)\right\|=\left\|\hat{p}_{i}+t\left(V_{i}-V_{j}\right)-\hat{p}_{j}\right\| \quad \forall t \in[0, T], \forall i, j \in \mathcal{A},
$$


provides an equivalent condition to (1). Namely, to ensure separation between $i$ and $j$, one can consider that $i$ flies at the relative velocity given by $V_{i j}:=V_{i}-V_{j}$ and that $j$ remains still. That is, (1) can be rewritten into the following equivalent condition:

$$
\left\|\hat{p}_{i}+t V_{i j}-\hat{p}_{j}\right\| \geq d \quad \forall t \in[0, T], \forall i, j \in \mathcal{A} .
$$

Figure 2 shows a geometrical analysis of the conflict between two aircraft $i$ and $j$ based on (2). When $V_{i j}$ points somewhere inside the disk of radius $d$ and center $\hat{p}_{j}, D\left(\hat{p}_{j}, d\right),(2)$ is not satisfied and vice versa. To avoid the conflict, $V_{i}$ and $V_{j}$ must be adjusted so that the new relative vector, $V_{i j}$, lies outside the disk. The two tangents to $D\left(\hat{p}_{j}, d\right)$ passing through $\hat{p}_{i}$, which are depicted on dashed-dotted lines in the figure, mark the limits for such a suitable vector $V_{i j}$.

In order to give a mathematical expression of aircraft separation according to this new interpretation, we will focus on the angles depicted on Figure 2. First, let us consider the segment joining $\hat{p}_{i}$ and $\hat{p}_{j}$, whose slope, measured from the $x$-axis and counter-clockwise, we denote by $\beta_{i j}$. Then, the slopes of the mentioned tangents can be obtained by adding to and subtracting from $\beta_{i j}$ a same angle $\alpha_{i j}$. If we call $\phi_{i j}$ the heading angle of the relative vector of velocity $V_{i j}$, it is sufficient to impose that either $\phi_{i j}$ is greater than $\beta_{i j}+\alpha_{i j}$ or smaller than $\beta_{i j}-\alpha_{i j}$ to guarantee the desired separation. That is, assuming that all angles belong to the interval $[0,2 \pi]$ and are measured counter-clockwise from the $x$-axis, the following constraint is equivalent to $(2)$

$$
\forall i, j \in \mathcal{A}: \quad \phi_{i j} \geq \beta_{i j}+\alpha_{i j} \quad \text { or } \quad \phi_{i j} \leq \beta_{i j}-\alpha_{i j} .
$$

Note that both $\beta_{i j}$ and $\alpha_{i j}$ only depend on the problem input, i.e., they should be considered as problem data. The only variable in the new separation condition is then $\phi_{i j}$. In order to find a mathematical formula to represent $\alpha_{i j}$, we shall refer again to Figure 2. We observe here that each tangent is the hypotenuse of a right triangle formed by the corresponding perpendicular radial segment in $D\left(\hat{p}_{j}, d\right)$ and the segment that links $\hat{p}_{i}$ to $\hat{p}_{j}$. Using then trigonometric relations, the following expression for $\alpha_{i j}$ is obtained

$$
\alpha_{i j}:=\arcsin \left(\frac{d}{\hat{d}_{i j}}\right),
$$

where $\hat{d}_{i j}$ is the distance between the initial positions $\hat{p}_{i}$ and $\hat{p}_{j}$.

Several authors have used different versions of (3) in their models. In the following, we describe their separation conditions by chronological order of paper publication. 


\subsubsection{Bilimoria's equations.}

To the best of our knowledge, Bilimoria was the first one to suggest this geometrical interpretation of conflicts, see Bilimoria (2000). He proposed closed-form expressions oriented to correct relative vectors heading so that they satisfy (3). As starting point, he supposed that one of the constraints in (3) was going to be satisfied as equality for every pair of aircraft in conflict. That is, for $i, j \in \mathcal{A}$ in conflict, the author set $\phi_{i j}=\beta_{i j} \pm \alpha_{i j}$. This means that such pairs attain horizontal separation equal to the safety distance $d$ in the solution. The author argued that other change in $\phi_{i j}$ would not be "efficient", since the objective was to minimize deviation from nominal paths (note that this approach is oriented to address single conflicts only). Once

$\phi_{i j}$ was fixed, the following chain of identities helped the author to derived his closed-form equations:

$$
\begin{aligned}
\frac{\sin \left(\phi_{i j}\right)}{\cos \left(\phi_{i j}\right)} & =\frac{v_{i} \sin \theta_{i}-v_{j} \sin \theta_{j}}{v_{i} \cos \theta_{i}-v_{j} \cos \theta_{j}} \\
\sin \left(\phi_{i j}\right)\left(v_{i} \cos \theta_{i}-v_{j} \cos \theta_{j}\right) & =\cos \left(\phi_{i j}\right)\left(v_{i} \sin \theta_{i}-v_{j} \sin \theta_{j}\right) \\
v_{i} \sin \left(\phi_{i j}-\theta_{i}\right) & =v_{j} \sin \left(\phi_{i j}-\theta_{j}\right) .
\end{aligned}
$$

Bilimoria addressed the three main variants of the CDR: SC, HAC, and SHAC. In the first two cases, expressions for $v_{i}$ and $\theta_{i}$ were obtained respectively from (4c), assuming that $i$ is the only aircraft in the conflicting pair that makes the correcting maneuvers:

$$
v_{i}=v_{j}\left(\frac{\sin \left(\phi_{i j}-\hat{\theta}_{j}\right)}{\sin \left(\phi_{i j}-\hat{\theta}_{i}\right)}\right), \quad \theta_{i}=\phi_{i j}-\arcsin \left(\frac{\hat{v}_{j}}{\hat{v}_{i}} \sin \left(\phi_{i j}-\theta_{j}\right)\right) .
$$

Some additional considerations that we omit here, such as the objective function of the problem and some trigonometric relations, were used by the author to derive the equations for the SHAC variant. For multiple-aircraft conflicts the proposed approach was to perform several cycles to resolve them sequentially by pairs. As the author reported himself, such strategy is not optimal.

\subsubsection{Pallottino et al.'s equations.}

A second work that relied on the presented geometrical characterization of conflicts is that of Pallottino et al. (2002). In this case, the authors proposed two MILPs for SC and HAC, respectively. These were one of the first mathematical programming formulations of the CDR in the literature. 


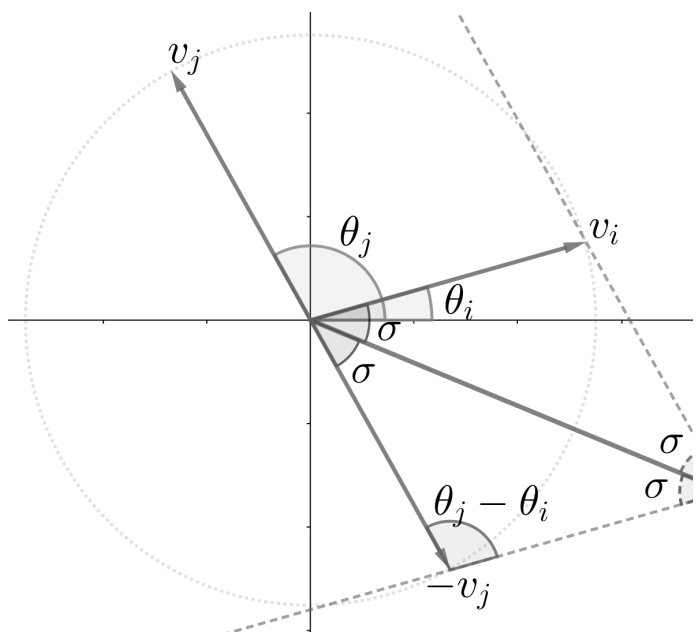

(a) If $v_{i}=v_{j}$ : Yes

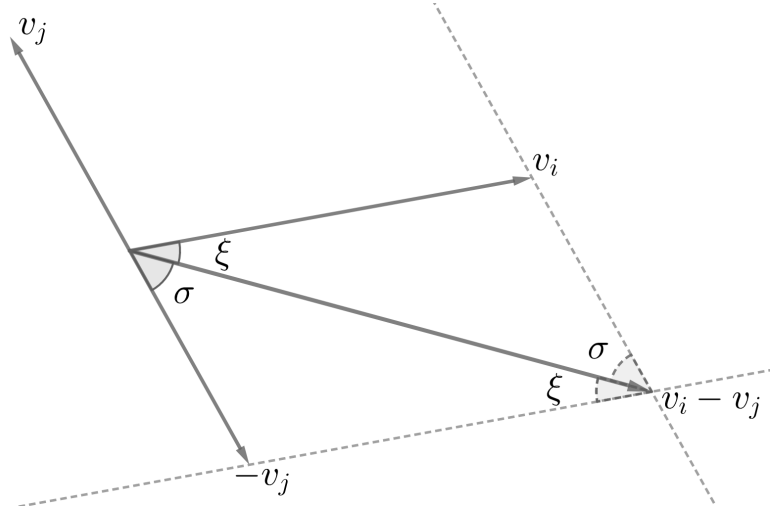

(b) If $v_{i} \neq v_{j}$ : No

Figure 3: Can be $\phi_{i j}$ expressed in terms of $\theta_{i}$ and $\theta_{j}$ ?

The authors introduced different separation conditions for each version of the problem, which were linear respectively on aircraft speeds and heading angles. The separation constraints for the SC variant read as follows

$\forall i, j \in \mathcal{A}: \quad \frac{v_{i} \sin \theta_{i}-v_{j} \sin \theta_{j}}{v_{i} \cos \theta_{i}-v_{j} \cos \theta_{j}} \geq \tan \left(\beta_{i j}+\alpha_{i j}\right) \quad$ or $\quad \frac{v_{i} \sin \theta_{i}-v_{j} \sin \theta_{j}}{v_{i} \cos \theta_{i}-v_{j} \cos \theta_{j}} \leq \tan \left(\beta_{i j}-\alpha_{i j}\right)$.

Note that (6) is equivalent to taking tangents on the inequalities of (3). The new inequalities were linearized by the authors by introducing binary variables to indicate the sign of the denominators. Regarding HAC, Pallottino et al. proposed a second approach to aircraft deconfliction. In their framework, the authors do not only consider constant aircraft speed, but also suppose speed to be the same for all aircraft. For a given pair $i, j \in \mathcal{A}$, they define the bisector of the sum of their heading angles, i.e., the line with slope $\frac{\theta_{i}+\theta_{j}}{2}$, and the orthogonal to the bisector, with slope $\frac{\theta_{i}+\theta_{j}+\pi}{2}$. They argue then that

$$
\forall i, j \in \mathcal{A}: \quad \frac{\theta_{i}+\theta_{j}+\pi}{2} \geq \beta_{i j}+\alpha_{i j} \quad \text { or } \quad \frac{\theta_{i}+\theta_{j}+\pi}{2} \leq \beta_{i j}-\alpha_{i j}
$$

is a separation condition for the HAC variant, where angles are taken in $[-\pi, \pi]$. It is easy to see that (7) is equivalent to (3) if all aircraft fly at the same speed. Indeed, the parallelogram representing the sum of the vectors $V_{i}$ and $-V_{j}$ is a rhombus in such a case (see Figure 3a). As illustrated by 
the figure, a diagonal of the rhombus bisects its interior angle. It can be proven that $\phi_{i j}=\frac{\theta_{i}+\theta_{j}+\pi}{2}+\pi$ by using a basic geometrical argument on the same figure (namely that $\phi_{i j}=\theta_{j}+\pi+\sigma$ and $\pi=\theta_{j}-\theta_{i}+2 \sigma$ ). The shift $\pi$ between the two formulas, (7) and (3), is due to a change in the angles domain. Note that this equivalence only stands if the magnitude of the velocity vectors coincide (see Figure $3 \mathrm{~b}$ for a counter-example).

After conditions (6) and (7) were proposed in Pallottino et al. (2002), they have been used by other authors, see, for instance, Alonso-Ayuso et al. (2010, 2014, 2016).

\subsubsection{Vela et al.'s equations.}

Finally, Vela et al. (2010) derive their own separation conditions in a similar way to Pallottino et al. (2002). We do not report here their equations since they are indeed the same as (6), except that the tangent functions of the right hand sides of the disjunctive constraints are substituted by their values at the given angles. Indeed, this is proven in Section 3.4, where $\tan \left(\beta_{i j}+\alpha_{i j}\right)$ and $\tan \left(\beta_{i j}-\alpha_{i j}\right)$ are explicitly calculated.

\subsection{Analytical conditions}

A different way of obtaining mathematical programming constraints for separation is to apply some analytical calculus on (1). The idea is to find, for each pair of aircraft, the critical time instant at which this constraint has to be satisfied. Since distances are positive, we base our analysis on the squared version of (1).

\subsubsection{Minimum-distance time equations.}

Given a pair of aircraft $i, j \in \mathcal{A}$, let $f_{i j}(t):=\left\|p_{i}(t)-p_{j}(t)\right\|^{2}$ represent the squared distance between them as a function of time. By assuming a rectilinear motion of the aircraft, the following expression yields

$$
f_{i j}(t)=\left\|V_{i j}\right\|^{2} t^{2}+2 t\left\langle\hat{p}_{i j}, V_{i j}\right\rangle+\hat{d}_{i j}^{2} \quad \forall t \in[0, T],
$$

where we have used the fact that $\hat{d}_{i j}=\left\|\hat{p}_{i j}\right\|$. It is now possible to calculate the time instant at which minimum separation between aircraft $i$ and $j$ is attained, $t_{i j}^{\min }$. Indeed, by calculating the first and second order derivatives of $f_{i j}(t)$, it can be easily seen that

$$
t_{i j}^{m i n}=\frac{-\left\langle\hat{p}_{i j}, V_{i j}\right\rangle}{\left\|V_{i j}\right\|^{2}}
$$


If $t_{i j}^{\text {min }}<0$, the minimum separation between $i$ and $j$ was attained in the past, and their trajectories are diverging in the given time horizon. Otherwise, by substituting $t_{i j}^{\text {min }}$ in (8), we obtain the minimum squared distance between $i$ and $j$ during their observed trajectories,

$$
f_{i j}^{\min }=\frac{-\left\langle\hat{p}_{i j}, V_{i j}\right\rangle^{2}}{\left\|V_{i j}\right\|^{2}}+\hat{d}_{i j}^{2}
$$

A new separation condition, also equivalent to (1), can be thus stated as follows,

$$
\forall i, j \in \mathcal{A}: \quad t_{i j}^{\min }<0 \quad \text { or } \quad f_{i j}^{\text {min }} \geq d^{2} .
$$

Some authors used this alternative separation condition in their mathematical programming models for the CDR, see for instance Cafieri \& Durand (2014), Cafieri \& D'Ambrosio (2018). As we discuss in Section 4, binary variables are typically considered to model the disjunction in (11). Observe that (11), unlike the original separation equation (1), does not depend on time, which solves the main obstacle for mathematically formulating the problem. The possibilities of analytical calculus on distance-related functions, however, does not end here.

Before continuing, let us write an extended version of (11),

$$
\forall i, j \in \mathcal{A}: \quad t_{i j}^{m i n}<0 \quad \text { or } \quad\left\|V_{i j}\right\|^{2}\left(\hat{d}_{i j}^{2}-d^{2}\right)-\left\langle\hat{p}_{i j}, V_{i j}\right\rangle^{2} \geq 0,
$$

and consider the following function

$$
g\left(V_{x i j}, V_{y i j}\right):=V_{x i j}^{2}\left(\hat{y}_{i j}^{2}-d^{2}\right)+V_{y i j}{ }^{2}\left(\hat{x}_{i j}^{2}-d^{2}\right)-2 V_{x i j} V_{y i j} \hat{x}_{i j} \hat{y}_{i j},
$$

which coincides with the scalar form of the left-hand side of the inequality in the second disjunctive term in (12).

\subsubsection{Rey ES Hijazi 's disjunctive equations.}

Rey \& Hijazi (2017b) studied the sign of $g$ to characterize the regions of the space of relative velocities $\left(V_{x i j}, V_{y i j}\right)$ in which (12) was satisfied. To that end, the authors study the roots of $g$ by treating either $V_{y i j}$ or $V_{x i j}$ as a constant. The discriminants of the resulting univariate quadratic function are, respectively:

$$
\Delta_{x i j}=4 d^{2} V_{y i j}{ }^{2}\left(\hat{x}_{i j}^{2}+\hat{y}_{i j}^{2}-d^{2}\right) \quad \text { and } \quad \Delta_{y i j}=4 d^{2} V_{x i j}{ }^{2}\left(\hat{x}_{i j}^{2}+\hat{y}_{i j}^{2}-d^{2}\right),
$$

and the roots are:

$$
V_{x i j}=\frac{2 \hat{x}_{i j} \hat{y}_{i j} \pm \sqrt{\Delta_{x i j}}}{2\left(\hat{y}_{i j}^{2}-d^{2}\right)} \quad \text { and } \quad V_{y i j}=\frac{2 \hat{x}_{i j} \hat{y}_{i j} \pm \sqrt{\Delta_{y i j}}}{2\left(\hat{x}_{i j}^{2}-d^{2}\right)} .
$$




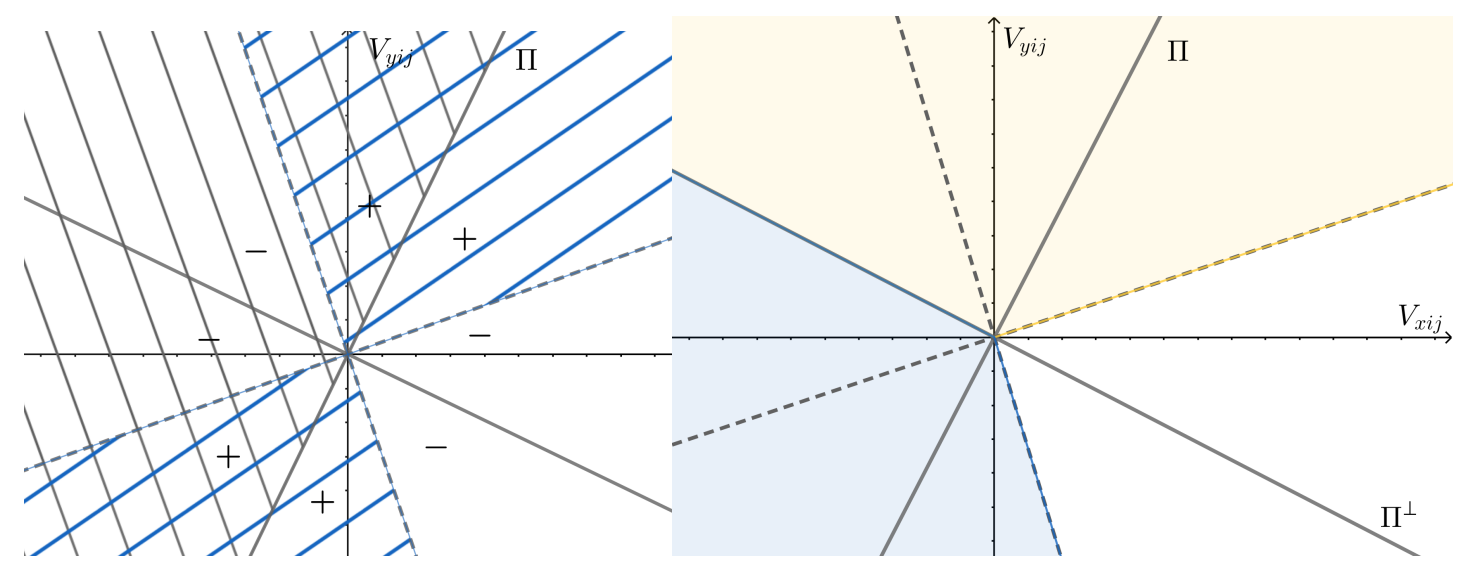

$\begin{array}{ll}\text { (a) Sign of } g\left(V_{x i j}, V_{y i j}\right) & \text { (b) Non-convex disjunctive feasible re- }\end{array}$ gions

Figure 4: Regions of the space $\left(V_{x i j}, V_{y i j}\right)$ delimited by (13), (14), $\Pi$ and $\Pi^{\perp}$

The authors noted that these roots always exist since the discriminants are positive because $\hat{x}_{i j}^{2}+\hat{y}_{i j}^{2}-d^{2} \geq 0$ (aircraft are assumed to be initially separated). Then, substituting the discriminants in the equations of the roots, they conclude that the points satisfying $g\left(V_{x i j}, V_{y i j}\right)=0$ satisfy one of the following equations

$$
\begin{aligned}
& \left(\hat{y}_{i j}^{2}-d^{2}\right) V_{x i j}-\left(\hat{x}_{i j} \hat{y}_{i j}+d \sqrt{\hat{x}_{i j}^{2}+\hat{y}_{i j}^{2}-d^{2}}\right) V_{y i j}=0 \\
& \left(\hat{y}_{i j}^{2}-d^{2}\right) V_{x i j}-\left(\hat{x}_{i j} \hat{y}_{i j}-d \sqrt{\hat{x}_{i j}^{2}+\hat{y}_{i j}^{2}-d^{2}}\right) V_{y i j}=0 \\
& \left(\hat{x}_{i j}^{2}-d^{2}\right) V_{y i j}-\left(\hat{x}_{i j} \hat{y}_{i j}-d \sqrt{\hat{x}_{i j}^{2}+\hat{y}_{i j}^{2}-d^{2}}\right) V_{x i j}=0 \\
& \left(\hat{x}_{i j}^{2}-d^{2}\right) V_{y i j}-\left(\hat{x}_{i j} \hat{y}_{i j}+d \sqrt{\hat{x}_{i j}^{2}+\hat{y}_{i j}^{2}-d^{2}}\right) V_{x i j}=0 .
\end{aligned}
$$

It is easy to observe that (13) and (15) define an identical line in the space $\left(V_{x i j}, V_{y i j}\right)$ (their coefficients are proportional). Similarly, (14) and (16) also stand for a same line. The problem remains then to identify in which portions of the space delimited by those lines $g\left(V_{x i j}, V_{y i j}\right) \geq 0$ holds.

Rey \& Hijazi considered $\Pi \equiv V_{x i j} \hat{x}_{i j}+V_{y i j} \hat{y}_{i j}=0$, the plane equation of the dot product $\left\langle\hat{p}_{i j}, V_{i j}\right\rangle$. They defined two sub-spaces,

$\left\{\left(V_{x i j}, V_{y i j}\right): V_{y i j} \hat{x}_{i j}-V_{x i j} \hat{y}_{i j} \leq 0\right\} \quad$ and $\quad\left\{\left(V_{x i j}, V_{y i j}\right): V_{y i j} \hat{x}_{i j}-V_{x i j} \hat{y}_{i j} \geq 0\right\}$,

induced by the plane perpendicular to $\Pi, \Pi^{\perp}$. As proven in Dias et al. (2020), $\Pi$ and $\Pi^{\perp}$ are bisectors of the angles formed by lines (13) and (14). 
Moreover, Dias et al. proved that $g$ is negative on the points of $\Pi^{\perp}$, which allowed them to classify the space as desired. Figure 4 a gives an illustration of the resulting partition in sub-spaces and the sign of $g$ in each of them. Dashed lines represent (13) and (14) and blue hashed sub-spaces define the disjunctive region where $g$ is positive. Grey hashed sub-space corresponds to diverging trajectories, while the blank area matches the unfeasible ones. Figure $4 \mathrm{~b}$ illustrates the feasible region of the space of relative velocities, i.e., the region in which either $g$ is positive or the trajectories diverge. Rey $\&$ Hijazi identified such non-convex region based on the sign of $\hat{x}_{i j}$ and $\hat{y}_{i j}$, as follows:

1. If $V_{y i j} \hat{x}_{i j}-V_{x i j} \hat{y}_{i j} \geq 0$, the formulas are derived from (13) and (15):

- Case $\hat{x}_{i j} \geq 0$ and $\hat{y}_{i j}<0:\left(\hat{x}_{i j}^{2}-d^{2}\right) V_{y i j}-\left(\hat{x}_{i j} \hat{y}_{i j}-d \sqrt{\hat{x}_{i j}^{2}+\hat{y}_{i j}^{2}-d^{2}}\right) V_{x i j} \geq$ 0

- Case $\hat{x}_{i j}<0$ and $\hat{y}_{i j} \geq 0:\left(\hat{x}_{i j}^{2}-d^{2}\right) V_{y i j}-\left(\hat{x}_{i j} \hat{y}_{i j}-d \sqrt{\hat{x}_{i j}^{2}+\hat{y}_{i j}^{2}-d^{2}}\right) V_{x i j} \leq$ 0

- Case $\hat{x}_{i j} \geq 0$ and $\hat{y}_{i j} \geq 0$ : $\left(\hat{y}_{i j}^{2}-d^{2}\right) V_{x i j}-\left(\hat{x}_{i j} \hat{y}_{i j}+d \sqrt{\hat{x}_{i j}^{2}+\hat{y}_{i j}^{2}-d^{2}}\right) V_{y i j} \leq$ 0

- Case $\hat{x}_{i j}<0$ and $\hat{y}_{i j}<0:\left(\hat{y}_{i j}^{2}-d^{2}\right) V_{x i j}-\left(\hat{x}_{i j} \hat{y}_{i j}+d \sqrt{\hat{x}_{i j}^{2}+\hat{y}_{i j}^{2}-d^{2}}\right) V_{y i j} \geq$ 0

2. If $V_{y i j} \hat{x}_{i j}-V_{x i j} \hat{y}_{i j} \leq 0$, the formulas are obtained from (14) and (16):

- Case $\hat{x}_{i j} \geq 0$ and $\hat{y}_{i j}<0:\left(\hat{y}_{i j}^{2}-d^{2}\right) V_{x i j}-\left(\hat{x}_{i j} \hat{y}_{i j}-d \sqrt{\hat{x}_{i j}^{2}+\hat{y}_{i j}^{2}-d^{2}}\right) V_{y i j} \leq$ 0

- Case $\hat{x}_{i j}<0$ and $\hat{y}_{i j} \geq 0:\left(\hat{y}_{i j}^{2}-d^{2}\right) V_{x i j}-\left(\hat{x}_{i j} \hat{y}_{i j}-d \sqrt{\hat{x}_{i j}^{2}+\hat{y}_{i j}^{2}-d^{2}}\right) V_{y i j} \geq$ 0

- Case $\hat{x}_{i j} \geq 0$ and $\hat{y}_{i j} \geq 0:\left(\hat{x}_{i j}^{2}-d^{2}\right) V_{y i j}-\left(\hat{x}_{i j} \hat{y}_{i j}+d \sqrt{\hat{x}_{i j}^{2}+\hat{y}_{i j}^{2}-d^{2}}\right) V_{x i j} \leq$ 0

- Case $\hat{x}_{i j}<0$ and $\hat{y}_{i j}<0:\left(\hat{x}_{i j}^{2}-d^{2}\right) V_{y i j}-\left(\hat{x}_{i j} \hat{y}_{i j}+d \sqrt{\hat{x}_{i j}^{2}+\hat{y}_{i j}^{2}-d^{2}}\right) V_{x i j} \geq$ 0

Each of the descriptions 1 and 2 corresponds to one of the sub-spaces yellow and blue in Figure 4b. To find the correct sign of the inequalities like in the above case analysis, it is sufficient to evaluate (13)-(16) on a point of $\Pi^{\perp}$, for instance $\left(-\hat{x}_{i j},-\hat{y}_{i j}\right)$.

Now, separation condition (12) can be expressed based on the above analysis. To this end, let us synthesize it by considering the separation 
inequalities $h_{1}\left(V_{x i j}, V_{y i j}\right) \leq 0$ and $h_{2}\left(V_{x i j}, V_{y i j}\right) \leq 0$, with $h_{1}$ and $h_{2}$ having the right coefficients depending on the analysis made on cases 1 and 2 above. The following is then a separation condition for the CDR,

$$
\begin{aligned}
& \forall i, j \in \mathcal{A}: \quad\left(V_{y i j} \hat{x}_{i j}-V_{x i j} \hat{y}_{i j} \geq 0 \quad \text { and } \quad h_{1}\left(V_{x i j}, V_{y i j}\right) \leq 0\right) \quad \text { or } \\
&\left(V_{y i j} \hat{x}_{i j}-V_{x i j} \hat{y}_{i j} \leq 0 \quad \text { and } \quad h_{2}\left(V_{x i j}, V_{y i j}\right) \leq 0\right) .
\end{aligned}
$$

Rey \& Hijazi and Dias et al. used these disjunctive conditions to build their models. Note that the gain with respect to (12) is not negligible: the new equations are linear in $V_{i j}$.

\subsection{Crossing point conditions}

A third alternative to model aircraft separation (1) is to develop a geometrical analysis of conflicts based on aircraft encounter. This approach builds on the fact that conflicts may only occur around the crossing points of aircraft pairs trajectories. The main assumption so that separation is ensured is that aircraft trajectories are straight within this region (although they may include turns before and after it). Let $i$ and $j$ be two aircraft with co-planar crossing trajectories, and let $\gamma_{i j}$ be their confluence angle, $\gamma_{i j} \in[0, \pi]$. After squaring condition (1), it can be rewritten as follows

$\left\|p_{i}(t)\right\|^{2}+\left\|p_{j}(t)\right\|^{2}-2\left\|p_{i}(t)\right\| \cdot\left\|p_{j}(t)\right\| \cdot \cos \gamma_{i j} \geq d^{2} \quad \forall t \in[0, T], \forall i, j \in \mathcal{A}$.

\subsubsection{Irvine's equations.}

Equation (18) was first suggested in Irvine (2001, 2002). The author considers the two dimensional space of aircraft positions to have its origin at the crossing point of the trajectories of $i$ and $j$. Then, (18) describes an ellipse in the two dimensional space given by $\left(\left\|p_{i}(t)\right\|,\left\|p_{j}(t)\right\|\right)$, i.e., the vector of distances between $i$ and $j$ and the crossing point of their trajectories, at time $t$. Whether or not a conflict will occur, depends upon whether the line defined by the points $\left(\left\|p_{i}(t)\right\|,\left\|p_{j}(t)\right\|\right)$ passes through the ellipse. According to Irvine, the tangents to the ellipse have equations

$$
y=\frac{v_{j}}{v_{i}} x \pm \frac{d \sqrt{\frac{v_{j}^{2}}{v_{i}^{2}}-2 \frac{v_{j}}{v_{i}} \cos \gamma_{i j}+1}}{\sin \gamma_{i j}},
$$

thus being the following a separation condition

$$
\left|\left\|p_{j}(t)\right\|-\frac{v_{j}}{v_{i}}\left\|p_{i}(t)\right\|\right| \geq \frac{d \sqrt{\frac{v_{j}^{2}}{v_{i}^{2}}-2 \frac{v_{j}}{v_{i}} \cos \gamma_{i j}+1}}{\sin \gamma_{i j}} \quad \forall t \in[0, T] .
$$




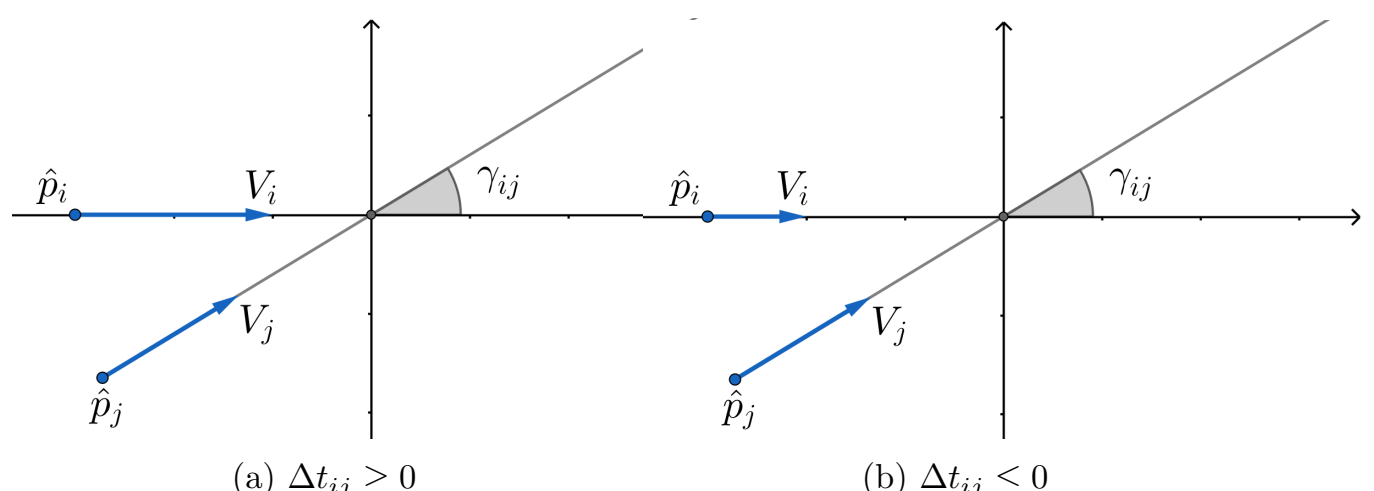

Figure 5: Trajectories meeting at the origin

The author then relies on (19) to discuss conflict probability estimation under the so-called along-track and cross-track errors. We refer the interested reader to Irvine (2002) for further details since the discussion on uncertainty is out of the scope of this paper. However, we notice that the analysis of Irvine of conflict probability inspired later works, including Vela et al. (2009a), Lehouillier et al. (2017a), which are mentioned in Section 4.

\subsubsection{Carlier et al.'s equations.}

As for Irvine's equations, we assume here that the trajectories of $i$ and $j$ meet at the origin. In addition, supposing without loss of generality that the trajectory of $i$ coincides with the $x$-axis, the following equations describe the motion of the aircraft,

$$
p_{i}(t)=t\left(v_{i}, 0\right), \quad p_{j}(t)=\left(t-\Delta t_{i j}\right)\left(v_{j} \cos \gamma_{i j}, v_{j} \sin \gamma_{i j}\right),
$$

where $\Delta t_{i j}$ is the time difference between the crossing of the origin by $i$ and $j$. Figure 5 illustrates aircraft trajectories under this configuration. In principle, one can think in $\Delta t_{i j}$ as a positive or negative value (see, respectively, left and right hand sides of the figure). Note that motion equations (20) involve a shift in the time horizon, which now start from negative instant times. The following example further illustrates the equations.

Example 1. Figure 5 shows an example of two crossing trajectories illustrating equations (20). Marks on the axes stand for 0.5 units steps.

Figure $5 a$ on the left shows the trajectories of aircraft $i$ and $j$ crossing at the origin, being $i$ the first one traversing the crossing point $\left(\Delta t_{i j} \geq 0\right)$. Conversely, Figure $5 b$ on the right shows a similar scenario where $j$ traverses the crossing point before $i\left(\Delta t_{i j} \leq 0\right)$. 
The equations of the trajectories depicted on Figure 5a are

$p_{i}(t)=t(1,0), \quad p_{j}(t)=(t-0.5)(0.8 \cos 0.54,0.8 \sin 0.54) \quad t \in[-1.5, T-1.5]$.

Note that, due to the equation of $p_{i}$ in (20), this aircraft always traverses the origin at $t=0$, which yields a shift in the considered time horizon equal to $\hat{x}_{i} / v_{i}$. In the scenario depicted by Figure $5 a$, the resulting time window is $[-1.5, T-1.5]$. At the beginning of this time interval, the initial positions of the aircraft are

$p_{i}(-1.5)=(-1.5,0) ; \quad$ and $\quad p_{j}(-1.5)=-2(0.8 \cos 0.54,0.8 \sin 0.54)=(-1.36,-0.83)$.

They cross the origin at time instants 0 and 0.5 respectively, being $\Delta t_{i j}=$ $0.5 \geq 0$.

On the other hand, the equations of aircraft trajectories of Figure $5 b$ are $p_{i}(t)=t(0.5,0) ; \quad$ and $\quad p_{j}(t)=(t+1)(0.8 \cos 0.54,0.8 \sin 0.54) \quad t \in[-3, T-3]$.

In this case, the aircraft cross the origin at $t=0$ and $t=-1$ respectively, being $\Delta t_{i j}=-1 \leq 0$.

If we substitute the new motion equations (20) in the encounter separation condition (18), we obtain the following inequalities:

$\left(v_{i}^{2}+v_{j}^{2}-2 v_{i} v_{j} \cos \gamma_{i j}\right) t^{2}-2 \Delta t_{i j} v_{j}\left(v_{j}-v_{i} \cos \gamma_{i j}\right) t+v_{j}^{2} \Delta t_{i j}^{2} \geq d^{2} \quad \forall t \in[0, T], \forall i, j \in \mathcal{A}$.

Following a similar argument than that of the beginning of Section 3.2, we consider the function of the squared distance between $i$ and $j$,

$f_{i j}(t)=\left(v_{i}^{2}+v_{j}^{2}-2 v_{i} v_{j} \cos \gamma_{i j}\right) t^{2}-2 \Delta t_{i j} v_{j}\left(v_{j}-v_{i} \cos \gamma_{i j}\right) t+v_{j}^{2} \Delta t_{i j}^{2} \quad \forall t \in[0, T]$.

It attains its minimum at

$$
t_{i j}^{\min }=\frac{\Delta t_{i j} v_{j}\left(v_{j}-v_{i} \cos \gamma_{i j}\right)}{v_{i}^{2}+v_{j}^{2}-2 v_{i} v_{j} \cos \gamma_{i j}},
$$

which can be substituted in (21) to obtain the minimum squared distance

$$
f_{i j}^{m i n}=\frac{-\left(\Delta t_{i j} v_{j}\left(v_{j}-v_{i} \cos \gamma_{i j}\right)\right)^{2}}{v_{i}^{2}+v_{j}^{2}-2 v_{i} v_{j} \cos \gamma_{i j}}+v_{j}^{2} \Delta t_{i j}^{2} .
$$

Here, we leave aside the question of the sign of $t_{i j}^{\text {min }}$, since we have shifted our time horizon. Then, a conflict occur (ignoring if it is being observed in our 
time window or not) if and only if $f_{i j}^{\min } \leq d^{2}$. Doing a few calculations, it is possible to isolate $\Delta t_{i j}$ in this expression to derive the following separation condition:

$$
\Delta t_{i j}^{2} \geq \frac{d^{2}\left(v_{i}^{2}+v_{j}^{2}-2 v_{i} v_{j} \cos \gamma_{i j}\right)}{v_{i}^{2} v_{j}^{2} \sin \gamma_{i j}^{2}} .
$$

It can be easily seen that (22) is equivalent to (19) after taking squares. Indeed, by multiplying (19) by $1 / v_{j}$, we obtain that its left-hand side coincides with $\Delta t_{i j}$ (due to the basic law by which space equals speed times time). On the other hand, the fact that the right-hand sides also match after the transformation is trivial.

In conclusion, if the pair $i, j$ satisfies condition (22), there is no conflict between them. But, what happens with multiple conflicts? We have assumed that the trajectories of a given pair meet at the origin, but this is surely not true when more than one conflicting aircraft are considered in the same scenario. On the contrary, (22) does not depend on the coordinate reference or time interval chosen at the beginning. Indeed, $\Delta t$ is an absolute component in our analysis since it represents a time lag. On the other hand, $\gamma_{i j}$, the confluence angle between trajectories, is independent from the coordinate axes chosen. Finally, since $v_{i}, v_{j}$, and $d$ are just scalar magnitudes, they are not affected by orientation. Consequently, (22) is a valid separation condition also for the multiple conflicts case.

Let us now resume the question of the sign of $\Delta t_{i j}$. According to the equations in (20), if $\Delta t_{i j} \geq 0, j$ traverses the origin (i.e., the crossing point of the trajectories) after $i$. Conversely, if $\Delta t_{i j} \leq 0, j$ is the first passing through the origin. Then, to simplify (22) by eliminating the powers, it is necessary to distinguish between two scenarios, namely, which aircraft traverses first the intersection point of the trajectories. Let us define the following function

$$
\Gamma\left(v_{i}, v_{j}, \gamma_{i j}\right)=\frac{d \cdot \sqrt{v_{i}^{2}+v_{j}^{2}-2 v_{i} v_{j} \cos \gamma_{i j}}}{v_{i} v_{j}\left|\sin \gamma_{i j}\right|},
$$

and denote with $t_{i}^{i \cap j}$ and $t_{j}^{i \cap j}$ the time instants in which $i$ and $j$ traverse the crossing point of their trajectories, respectively. The following is a separation condition for the CDR:

$$
\forall i, j \in \mathcal{A}: \quad t_{i}^{i \cap j}-t_{j}^{i \cap j} \geq \Gamma\left(v_{i}, v_{j}, \gamma_{i j}\right) \quad \text { or } \quad t_{j}^{i \cap j}-t_{i}^{i \cap j} \geq \Gamma\left(v_{i}, v_{j}, \gamma_{i j}\right) .
$$

The new separation condition does not seem particularly good. Like its analogous (12), it is nonlinear on aircraft velocity and, moreover, it involves 
trigonometric functions on the confluence angle (which would be a problem variable if heading angle changes are allowed). However, the potential of (23) is that it allows a new interpretation of the problem, focusing on its temporal component rather than on the spatial one.

Carlier et al. (2003) were the first to propose condition (23) and to interpret aircraft conflict resolution as a scheduling problem. In their proposed framework, aircraft trajectories were identified with jobs, and subtrajectories, which were determined by crossing points, were interpreted as the tasks of classical scheduling. Aircraft speeds can be recovered from time duration of tasks by using rectilinear motion laws. This pioneering work inspired other authors to develop their models, for instance, Vela et al. (2009b), Rey et al. (2014), Omer (2015), Rey et al. (2016), Courchelle et al. (2019). All of them approximate nonlinear expression in (23) by considering fixed values of aircraft speeds. This type of work usually considers that aircraft paths are sequences of waypoints, trajectories being piece-wise rectilinear. Separation conditions (23) are then imposed at common waypoints, under the assumption that the involved aircraft trajectories are rectilinear within a neighborhood of the conflict region. These models typically treat parallel trajectories separately, including the so-called trailing and frontal conflicts, since they are excluded from the analysis made to arrive at (23).

\subsection{Homogenizing separation}

Up to this point we have presented different ways of interpreting and representing the separation condition (1) with $T=+\infty$. Each of them look at the problem from a different perspective: some focus on its underlying geometry and other take an analytic view to obtain some critical points (in time or space) that characterize the conflicts. But, how are these approaches related? Are there so many different ways of imposing aircraft separation through mathematical equations? Or some of them are indeed the same? In this section, we try to answer these questions.

Something that the readers might have found noticeable is that equations in both (6) and (13)-(16) are linear functions of $V_{i j}$. It seems reasonable then to try to relate the coefficients in (13)-(16) to the tangent of the angles $\beta_{i j}+\alpha_{i j}$ and $\beta_{i j}-\alpha_{i j}$, which appeared in (6). Let us consider again $i, j \in \mathcal{A}$ two aircraft in conflict, $D\left(\hat{p}_{j}, d\right)$ the safety disk around $j$, and the tangents to $D\left(\hat{p}_{j}, d\right)$ that pass through $\hat{p}_{i}$. Any of these tangents, together with its corresponding perpendicular radius in the disk and the segment joining $\hat{p}_{i}$ and $\hat{p}_{j}$, form a right triangle (see illustration on Figure 6). The tangent of 


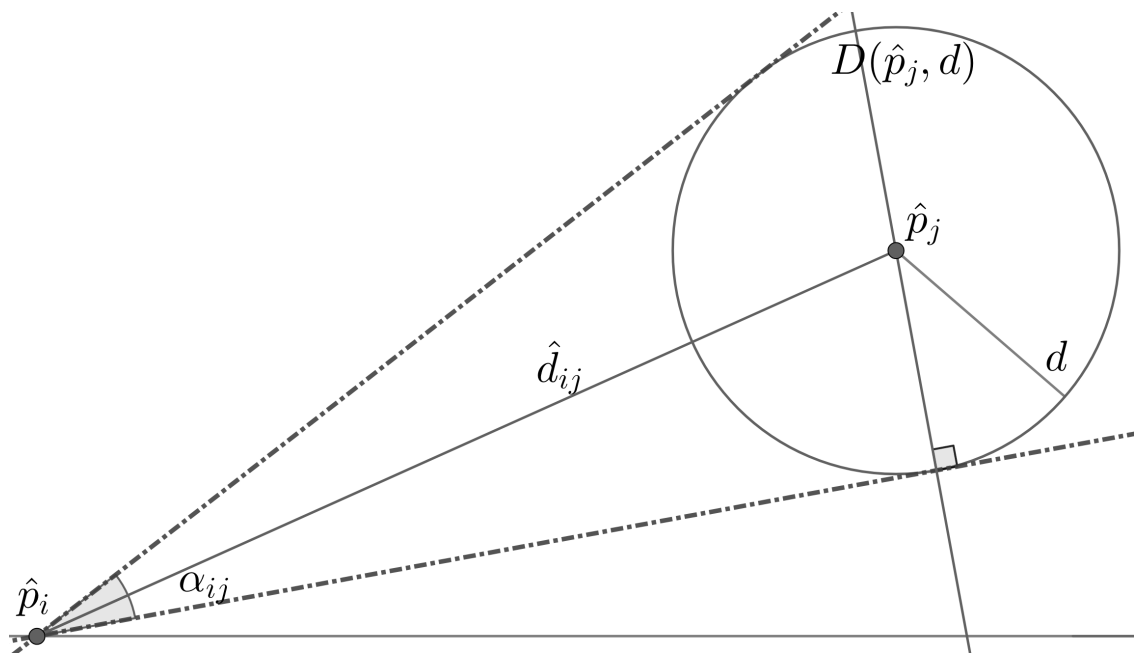

Figure 6: Illustration of geometrical calculation of $\tan \left(\alpha_{i j}\right)$

$\alpha_{i j}$ can be then expressed as follows

$$
\tan \alpha_{i j}=\frac{d}{\sqrt{\hat{d}_{i j}^{2}-d^{2}}} \equiv \tan \alpha_{i j}=\frac{d}{\sqrt{\hat{x}_{i j}^{2}+\hat{y}_{i j}^{2}-d^{2}}} .
$$

On the other hand, the tangent of $\beta_{i j}$ is, by definition,

$$
\tan \beta_{i j}=\frac{\hat{y}_{i j}}{\hat{x}_{i j}}
$$

By using trigonometric relations and arithmetic calculus we obtain then:

$$
\begin{aligned}
& \tan \left(\beta_{i j}+\alpha_{i j}\right)=\frac{\tan \beta_{i j}+\tan \alpha_{i j}}{1-\tan \beta_{i j} \tan \alpha_{i j}}=\frac{\hat{y}_{i j} \sqrt{\hat{x}_{i j}^{2}+\hat{y}_{i j}^{2}-d^{2}}+d \hat{x}_{i j}}{\hat{x}_{i j} \sqrt{\hat{x}_{i j}^{2}+\hat{y}_{i j}^{2}-d^{2}}-d \hat{y}_{i j}}, \\
& \tan \left(\beta_{i j}-\alpha_{i j}\right)=\frac{\tan \beta_{i j}-\tan \alpha_{i j}}{1+\tan \beta_{i j} \tan \alpha_{i j}}=\frac{\hat{y}_{i j} \sqrt{\hat{x}_{i j}^{2}+\hat{y}_{i j}^{2}-d^{2}}-d \hat{x}_{i j}}{\hat{x}_{i j} \sqrt{\hat{x}_{i j}^{2}+\hat{y}_{i j}^{2}-d^{2}}+d \hat{y}_{i j}} .
\end{aligned}
$$

If we multiply numerator and denominator by the "conjugate" of the denominator in each case, i.e., we multiply by $\hat{x}_{i j} \sqrt{\hat{x}_{i j}^{2}+\hat{y}_{i j}^{2}-d^{2}} \pm d \hat{y}_{i j}$, we 
obtain the following expressions:

$$
\begin{aligned}
\tan \left(\beta_{i j}+\alpha_{i j}\right) & =\frac{\hat{x}_{i j} \hat{y}_{i j}+d \sqrt{\hat{x}_{i j}^{2}+\hat{y}_{i j}^{2}-d^{2}}}{\hat{x}_{i j}^{2}-d^{2}}, \\
\tan \left(\beta_{i j}-\alpha_{i j}\right) & =\frac{\hat{x}_{i j} \hat{y}_{i j}-d \sqrt{\hat{x}_{i j}^{2}+\hat{y}_{i j}^{2}-d^{2}}}{\hat{x}_{i j}^{2}-d^{2}} .
\end{aligned}
$$

While, if we multiply numerator and denominator by the "conjugate" of the numerator in each case, i.e. $\hat{y}_{i j} \sqrt{\hat{x}_{i j}^{2}+\hat{y}_{i j}^{2}-d^{2}} \mp d \hat{x}_{i j}$, we obtain:

$$
\begin{aligned}
\tan \left(\beta_{i j}+\alpha_{i j}\right) & =\frac{\hat{y}_{i j}^{2}-d^{2}}{\hat{x}_{i j} \hat{y}_{i j}-d \sqrt{\hat{x}_{i j}^{2}+\hat{y}_{i j}^{2}-d^{2}}}, \\
\tan \left(\beta_{i j}-\alpha_{i j}\right) & =\frac{\hat{y}_{i j}^{2}-d^{2}}{\hat{x}_{i j} \hat{y}_{i j}+d \sqrt{\hat{x}_{i j}^{2}+\hat{y}_{i j}^{2}-d^{2}}}
\end{aligned}
$$

Observe now that, if we substitute the value of the tangents in (6) by the formulas in (24) and (25), we would obtain the same expressions as in (15) and (16). Similarly, (26) and (27) combined with (6) will yield the formulas in (13) and (14). As a consequence, the formulas yielding from the analytical calculus presented by Rey \& Hijazi are in essence equivalent reformulations of those derived by Pallottino et al. following a geometrical reasoning.

To summarize, we now know different equivalent separation conditions, namely six: (3), (6), (12), (17), (19) and (23)- (7) is excluded since it is only valid when all aircraft fly at the same speed. Some of them can be directly derived from each other, such as (3) and (6) (by taking tangents), (6) and (17) (as just shown), (19) and (23) (as shown in the previous subsection) or (12) and (23) (by changing spatial and temporal references). These equivalent separation conditions are expressed as functions of different elements of the problem such as aircraft velocities, angles, or even time. However, all of them have one thing in common: they are disjunctive conditions. This comes to show the combinatorial nature of the problem, which is not evident at first. As a consequence, existing mathematical programming formulations of the problem are for the most part mixed-integer, as we discuss in next section. 


\section{Mathematical programming formulations}

The CDR has been addressed from wide ranging domains, such as Optimal Control (see, e.g. Tarnopolskaya \& Fulton 2009), Simulation (see, e.g. Alliot et al. 1997), Visual Analytics (see, e.g. Zohrevandi et al. 2020) or Mathematical Programming (MP), in a heterogeneous effort to provide decision support tools for ATCs. In this section, we review some of the MP formulations proposed in the literature, aiming at providing an overview on the different existing kind of approaches. We suggest survey Kuchar \& Yang (2000) for an extended reading on other CDR methods. A classifying taxonomy is proposed therein, which includes dimension (vertical, horizontal, or $3 \mathrm{D}$ ), trajectory propagation model (straight, worst case, or probabilistic), conflict detection threshold (whether a model explicitly defines when a conflict alert is issued), conflict resolution method (prescribed maneuvers, optimization, force field, or manual), allowed maneuvers (speed, lateral, vertical, or combined) and multiple conflicts management (pairwise or global). To the best of our knowledge, there has not been a similar discussion focusing on comparison of MP approaches for CDR. In order to complete our exposition, which only consider a selection from the existing MP formulations, we have gathered those and other existing MP approaches on Table 1. They are chronologically displayed and classified based on different modelling aspects, which are described on the table foot. Our taxonomy includes the separation condition according to Section 3, which, to the best of our knowledge, has not been considered before to classify the models.

In MP formulations of CDR, the decision variables are usually the changes on the trajectories allowed, namely heading angle and/or speed changes. We focus on these two kinds of maneuvers. Other works also include variables to represent different altitude levels, in order to model flight level reallocation. This is frequently done with binary variables that activate or inactive separation constraints depending on whether a pair of aircraft fly at the same altitude or not.

Some works consider trajectory recovery of aircraft. This is more common when models are based on heading angle maneuvers since aircraft have to be returned to their original paths. There are roughly two kinds of strate-

gies to achieve trajectory recovery. One is to consider two optimization steps, the first for CDR and the second for recovery. Alternatively, some approaches consider both in the same optimization process, where maneuvers are considered to start and end at some time instants, and they finalize by going back to the original configuration.

In the following, we introduce some of the MP formulations in the lit- 
erature, classified depending on the allowed maneuvers. Due to the nature of the problem, many of them model logical constraints, for which binary variables are used. Here, we define common notation for these constraints for the sake of simplicity, which includes $z$-variables and big $M$. Of course, depending on the formulation at hand, they acquire different meanings. We also simplify constraints indexing by just considering pairs of aircraft as $i, j \in \mathcal{A}$, thus omitting condition $i<j$ for readability.

\subsection{Speed regulation}

Pallottino et al. (2002) suggest the use of binary variables to model their separation conditions (6) via MP. As a result, a MILP formulation of CDR based on SC can be obtained, as the authors indicate in their paper. Their decision variables are the increment or decrease of speeds $q_{i}^{\prime}$, where $v_{i}=\hat{v}_{i}+q_{i}^{\prime}$ for each aircraft $i \in \mathcal{A}$. Note that we use $q_{i}^{\prime}$ here in order to differentiate these variables from $q_{i}$, which were defined in Section 2.1 as the speed change ratio, $v_{i}=q_{i} \hat{v}_{i}$. Following the authors' indications, we reconstruct their formulation, where the short-hands $\vartheta_{i j}^{+}:=\beta_{i j}+\alpha_{i j}$ and $\vartheta_{i j}^{-}:=\beta_{i j}-\alpha_{i j}$ are used for readability:

$$
\begin{aligned}
& \min _{q^{\prime}, z} \quad \sum_{i \in \mathcal{A}}-q_{i}^{\prime} \\
& \text { s.t. } \quad \underline{q}_{i}^{\prime} \leq q_{i}^{\prime} \leq \bar{q}_{i}^{\prime} \\
& \left(\hat{v}_{j}+q_{j}^{\prime}\right) \cos \theta_{j}-\left(\hat{v}_{i}+q_{i}^{\prime}\right) \cos \theta_{i} \leq M\left(1-z_{i j}^{1}\right) \\
& \left(\hat{v}_{j}+q_{j}^{\prime}\right)\left(\sin \theta_{j}-\tan \vartheta_{i j}^{+} \cos \theta_{j}\right)-\left(\hat{v}_{i}+q_{i}^{\prime}\right)\left(\sin \theta_{i}-\tan \vartheta_{i j}^{+} \cos \theta_{i}\right) \leq M\left(1-z_{i j}^{1}\right) \\
& \left(\hat{v}_{j}+q_{j}^{\prime}\right) \cos \theta_{j}-\left(\hat{v}_{i}+q_{i}^{\prime}\right) \cos \theta_{i} \leq M\left(1-z_{i j}^{2}\right) \\
& \left(\hat{v}_{i}+q_{i}^{\prime}\right)\left(\sin \theta_{i}-\tan \vartheta_{i j}^{-} \cos \theta_{i}\right)-\left(\hat{v}_{j}+q_{j}^{\prime}\right)\left(\sin \theta_{j}-\tan \vartheta_{i j}^{-} \cos \theta_{j}\right) \leq M\left(1-z_{i j}^{2}\right) \\
& \left(\hat{v}_{i}+q_{i}^{\prime}\right) \cos \theta_{i}-\left(\hat{v}_{j}+q_{j}^{\prime}\right) \cos \theta_{j} \leq M\left(1-z_{i j}^{3}\right) \\
& \left(\hat{v}_{i}+q_{i}^{\prime}\right)\left(\sin \theta_{i}-\tan \vartheta_{i j}^{+} \cos \theta_{i}\right)-\left(\hat{v}_{j}+q_{j}^{\prime}\right)\left(\sin \theta_{j}-\tan \vartheta_{i j}^{+} \cos \theta_{j}\right) \leq M\left(1-z_{i j}^{3}\right) \\
& \left(\hat{v}_{i}+q_{i}^{\prime}\right) \cos \theta_{i}-\left(\hat{v}_{j}+q_{j}^{\prime}\right) \cos \theta_{j} \leq M\left(1-z_{i j}^{4}\right) \\
& \left(\hat{v}_{j}+q_{j}^{\prime}\right)\left(\sin \theta_{j}-\tan \vartheta_{i j}^{-} \cos \theta_{j}\right)-\left(\hat{v}_{i}+q_{i}^{\prime}\right)\left(\sin \theta_{i}-\tan \vartheta_{i j}^{-} \cos \theta_{i}\right) \leq M\left(1-z_{i j}^{4}\right) \\
& z_{i j}^{1}+z_{i j}^{2}+z_{i j}^{3}+z_{i j}^{4} \geq 1 \\
& z_{i j}^{1}, z_{i j}^{2}, z_{i j}^{3}, z_{i j}^{4} \in\{0,1\}
\end{aligned}
$$


The objective (28a) is to minimize the deceleration changes (i.e., negative values $\left.q_{i}^{\prime}\right)$. Constraints (28b) state the bounds on continuous $q^{\prime}$-variables. On the other hand, (28c)-(28j) model the disjunctive equation (6). They can be divided into two groups. Constraints (28c)-(28f) are a linearization of (6) when the sign of the denominator $v_{i} \cos \theta_{i}-v_{j} \cos \theta_{j}$ is positive (in this case at least one variable $z_{i j}^{1}$ or $z_{i j}^{2}$ is one), while (28g)-(28j) model (6) otherwise (in this case $z_{i j}^{3}$ or $z_{i j}^{4}$ is one). The constraints on these groups can be active, when the corresponding binary $z$-variable on the right-hand side takes value 1 , or inactive, if it takes value 0 . In the latter case, a large enough value of $M$ guarantees that the constraint does not modify the feasible region of the problem. Note that the same $M$ is used here in (28c)-(28j) for simplicity, but a different large enough constant can be used in each constraint. On the other hand, $(28 \mathrm{k})$ ensure that at least one pair of the constraints among (28c)-(28j) is active. Finally, constraints (281) state the binarity of $z$-variables.

Alonso-Ayuso et al. (2010) extended (28) by considering additional aspects including altitude level reallocation, trajectory recovery, and minimization of the number of maneuvers implemented per aircraft.

Cafieri \& Durand (2014) propose a MINLP based on separation condition (12), where speed regulation is performed at different time instants. While other models consider speed to be regulated at $t=0$ and maintained afterwards, Cafieri \& Durand define two time instants for each aircraft at which they start/end flying with modified speed. The result is a more flexible but more complex model, including products of continuous variables in the constraints. The objective function is to minimize deviation from the nominal plan. We refer the interested reader to the original paper since reproducing the formulation here would need from a significant amount of additional notation.

Cafieri \& Omheni (2017) present a MINLP, whose objective was to maximize the number of non-conflicting pairs via speed regulation. The authors use the following variables for each pair of aircraft $i, j \in \mathcal{A}$ :

$$
\eta_{i j}= \begin{cases}1, & \text { if } i \text { and } j \text { are separated } \\ 0, & \text { otherwise. }\end{cases}
$$

At the beginning of the time horizon, aircraft are assumed to be separated. Two cases are distinguished, namely whether aircraft trajectories are divergent or not. The formulation, which is based on analytical separation 
conditions (12), reads

$$
\begin{array}{ccc}
\max _{v, V, \eta, z} & \sum_{i \in \mathcal{A}} \eta_{i j} & \\
\text { s.t. } & \underline{v}_{i} \leq v_{i} \leq \bar{v}_{i} & \\
& V_{i}=\left(v_{i} \cos \hat{\theta}_{i}, v_{i} \sin \hat{\theta}_{i}\right) & \\
V_{i j}=V_{i}-V_{j} & \forall i \in \mathcal{A} & (29 \mathrm{a}) \\
\left(2 z_{i j}^{1}-1\right)\left(\left\|V_{i j}\right\|^{2}\left(\hat{d}_{i j}^{2}-d^{2}\right)-\left\langle\hat{p}_{i j}, V_{i j}\right)^{2}\right) \geq 0 & \forall i, j \in \mathcal{A} & (29 \mathrm{~d}) \\
\left(2 z_{i j}^{2}-1\right)\left\langle\hat{p}_{i j}, V_{i j}\right\rangle \geq 0 & \forall i, j \in \mathcal{A} & (29 \mathrm{e}) \\
\eta_{i j} \geq z_{i j}^{1} & \forall i, j \in \mathcal{A} & (29 \mathrm{f}) \\
\eta_{i j} \geq z_{i j}^{2} & \forall i, j \in \mathcal{A} & (29 \mathrm{~g}) \\
\eta_{i j} \leq z_{i j}^{1}+z_{i j}^{2} & \forall i, j \in \mathcal{A} & (29 \mathrm{~h}) \\
0 \leq \eta_{i j} \leq 1 & \forall i, j \in \mathcal{A} & (29 \mathrm{i}) \\
z_{i j}^{1}, z_{i j}^{2} \in\{0,1\} & \forall i, j \in \mathcal{A} . &
\end{array}
$$

Constraints (29a) limit velocity changes and (29b) and (29c) link scalar speeds to vectors of relative velocity. Constraints (29d) and (29e) ensure aircraft separation. When $z_{i j}^{1}=1,(29 \mathrm{~d})$ coincides with the right-hand side of disjunctive separation condition (12), and it ensures that $i$ and $j$ are separated at least the safety distance $d$. When $z_{i j}^{2}=1$, (29e) stands for the left-hand side of $(12)$, i.e., $t_{i j}^{\min }<0$, meaning that the trajectories of the aircraft are divergent. Due to the objective function and constraints (29h), for each pair of aircraft $i$ and $j$, at least one of the binaries $z_{i j}^{1}$ or $z_{i j}^{2}$ will take value one if feasible. At the same time, (29h) guarantees that $\eta_{i j}$ is zero if both $z_{i j}^{1}$ and $z_{i j}^{2}$ are. On the other hand, (29f) and (29g) enforce $\eta_{i j}$ to be one if either $z_{i j}^{1}$ or $z_{i j}^{2}$ are. Variables $\eta_{i j}$ will take values $0 / 1$ in the optimum, and that is why their binarity is relaxed in (29i).

Cafieri \& D'Ambrosio (2018) propose a MINLP in a similar vein. Inspired by Cafieri \& Durand (2014), they draw on the same analytical separation conditions, but they reformulate the model in order to isolate nonlinearities in the same constraints. This is interesting for specific solving techniques, such as the alternating heuristic they apply. Their reformula- 
tion reads

$$
\begin{array}{cc}
\sum_{\substack{q, V, W, s, S, z \\
\text { s.t. }}}\left(q_{i}-1\right)^{2} & \text { (30a) } \\
V_{x i j}=\hat{V}_{x i} q_{i}-\hat{V}_{x j} q_{j} ; \quad V_{y i j}=\hat{V}_{y i} q_{i}-\hat{V}_{y j} q_{j} & \forall i, j \in \mathcal{A}(30 \mathrm{~b}) \\
W_{x i j}=V_{x i j}^{2} ; \quad W_{y i j}=V_{y i j}^{2} & \forall i, j \in \mathcal{A}(30 \mathrm{c}) \\
s_{i j}=\hat{x}_{i j} V_{x i j}+\hat{y}_{i j} V_{y i j} & \forall i, j \in \mathcal{A}(30 \mathrm{~d}) \\
S_{i j}=s_{i j}^{2} & \forall i, j \in \mathcal{A}(30 \mathrm{e}) \\
X_{i j}=W_{x i j}+W_{y i j} & \forall i, j \in \mathcal{A}(30 \mathrm{f}) \\
\underline{s}_{i j} z_{i j} \leq s_{i j} \leq \bar{s}_{i j}\left(1-z_{i j}\right) & \forall i, j \in \mathcal{A}(30 \mathrm{~g}) \\
\left(\hat{d}_{i j}^{2}-d^{2}\right) X_{i j}-S_{i j} \geq M_{i j}\left(1-z_{i j}\right) & \forall i, j \in \mathcal{A}(30 \mathrm{~h}) \\
z_{i j} \in\{0,1\} & \forall i, j \in \mathcal{A} .
\end{array}
$$

The objective (30a) is to minimize the changes made on speed. From constraints (30b)-(30f), variables $s_{i j}$ stand for the scalar product $\left\langle\hat{p}_{i j}, V_{i j}\right\rangle$, while $X_{i j}$ represent the squared norm of the relative vector of velocity, $V_{i j}$. When $z_{i j}=1$, (30h) guarantees aircraft separation; when $z_{i j}=0$, (30g) states that the trajectories have to be divergent. Therefore, in this case, all the pairs have to be deconflicted: if this is not possible the problem becomes unfeasible.

Rey et al. (2014) present a MINLP to maximize the number of conflicts solved based on arrival times control. This represents the first stage of their proposed equity-oriented conflict resolution model, which is followed by two more, aimed at solving conflicts in the fairest way and reducing delay, respectively. Time is considered a function of the scalar speed, which is indirectly regulated by the model. Heading angle changes are not considered. Their MINLP is based on separation conditions (23). Instead of non-convex function $\Gamma\left(v_{i}, v_{j}, \gamma_{i j}\right)$, they use a linear approximation first introduced in their previous work, Rey et al. (2012). There, they define the following convex function

$$
\varphi_{i j}(r):=\sqrt{r^{2}-2 r \cos \gamma_{i j}+1}
$$

and noted that

$\Gamma\left(v_{i}, v_{j}, \gamma_{i j}\right)=\frac{d}{v_{i}\left|\sin \gamma_{i j}\right|} \varphi_{i j}\left(v_{i} / v_{j}\right) \quad$ and $\quad \Gamma\left(v_{i}, v_{j}, \gamma_{i j}\right)=\frac{d}{v_{j}\left|\sin \gamma_{i j}\right|} \varphi_{i j}\left(v_{j} / v_{i}\right)$. 
Maximum values of $\varphi_{i j}(r)$ are achieved for the maximum and minimum values of $r$. Thus, the authors defined

$$
\bar{\varphi}_{i j}:=\max \left\{\varphi\left(\underline{v}_{i} / \bar{v}_{j}\right), \varphi\left(\bar{v}_{i} / \underline{v}_{j}\right)\right\}, \quad \bar{\varphi}_{j i}:=\max \left\{\varphi\left(\underline{v}_{j} / \bar{v}_{i}\right), \varphi\left(\bar{v}_{j} / \underline{v}_{i}\right)\right\}
$$

(in the original paper only one value $\bar{\varphi}_{i j}$ is defined, a formal typo also made in Rey et al. (2016) that does not alter the final upper bound on $\Gamma$, Rey (2020)), which yield the following upper bounds on $\Gamma\left(v_{i}, v_{j}, \gamma_{i j}\right)$ :

$$
\frac{d}{v_{i}\left|\sin \gamma_{i j}\right|} \bar{\varphi}_{i j}, \quad \frac{d}{v_{j}\left|\sin \gamma_{i j}\right|} \bar{\varphi}_{j i} \text {. }
$$

The authors consider a path for each aircraft $i \in \mathcal{A}$, denoted by $\mathcal{P}(i)$ and made of a sequence of waypoints. Suppose that $k$ is the crossing point between the trajectories of $i, j \in \mathcal{A}$, that is, $\mathcal{P}(i)=\left\{\ldots, k_{i}^{-}, k, \ldots\right\}$ and $\mathcal{P}(j)=\left\{\ldots, k_{j}^{-}, k, \ldots\right\}$. If we denote by $t_{i}^{k}$ (resp. $t_{j}^{k}$ ) the time instant at which $i$ (resp. $j$ ) traverses the crossing point $k$, and by $t_{i}^{k^{-}}$(resp. $t_{j}^{k^{-}}$) the instant at which it traverses the preceding waypoint, the following upper bound on $\Gamma\left(v_{i}, v_{j}, \gamma_{i j}\right)$ is obtained from (31):

$$
\bar{\Gamma}_{i j}=\min \left\{\left(t_{i}^{k}-t_{i}^{k^{-}}\right) \frac{d \bar{\varphi}_{i j}}{d\left(k, k_{i}^{-}\right)\left|\sin \gamma_{i j}\right|},\left(t_{j}^{k}-t_{j}^{k^{-}}\right) \frac{d \bar{\varphi}_{j i}}{d\left(k, k_{j}^{-}\right)\left|\sin \gamma_{i j}\right|}\right\} .
$$

With $d(\cdot, \cdot)$ we denote the Euclidean distance. This upper bound is a linear function of the arrivals times, which are the only variables in the definition of $\bar{\Gamma}_{i j}$. Rey et al. (2014) use binary variables to represent effective separation between aircraft, an approach also adopted by Cafieri \& Omheni (2017). The definition changes slightly:

$\eta_{i j}= \begin{cases}1, & \text { if the conflict between } i \text { and } j, \text { with crossing trajectories, is solved } \\ 0, & \text { otherwise. }\end{cases}$

Using these variables, together with the arrival times to waypoints $t_{i}^{k}$, for each aircraft $i \in \mathcal{A}$ and waypoint $k \in \mathcal{P}(i)$, they proposed the following MINLP for the CDR:

$$
\begin{array}{cll}
\max _{t, \eta, \Omega} & \sum_{i, j \in \mathcal{A}} \eta_{i j} & \\
\text { s.t. } & \underline{t}_{i}^{k} \leq t_{i}^{k} \leq \bar{t}_{i}^{k} & \forall i \in \mathcal{A}, \forall k \in \mathcal{P}(i) \\
\Omega_{i, j}=\max \left\{0, \bar{\Gamma}_{i j}-\left|t_{i}^{k}-t_{j}^{k}\right|\right\} & \forall i, j \in \mathcal{A}, \forall k \in \mathcal{P}(i) \cap \mathcal{P}(j)(32) \\
\eta_{i j} \leq 1-\frac{\Omega_{i, j}}{M} & \forall i, j \in \mathcal{A} \\
\eta_{i j} \in\{0,1\} & \forall i, j \in \mathcal{A}
\end{array}
$$


The objective, (32a), is to maximize the number of solved crossing conflicts. Bounds on speed regulation are modelled in $(32 \mathrm{~b})$ through linear transformation based on aircraft rectilinear motion. Constraints (32c) ensure that $\Omega_{i, j}=0$ if and only if the conflict between $i$ and $j$ is solved. Because of (32d), if $\Omega_{i, j}>0$, the binary variable $\eta_{i j}$ will take value zero ( $M$ has to be large enough so that $\frac{\Omega_{i, j}}{M}<1$ ). Otherwise, it will take value one due to the maximizing objective. Constraints (32c) can be linearized by considering new binary variables and some additional constraints. For more details on this linearization, see the Appendix in Rey et al. (2014).

Finally, Cerulli et al. (2020) interpret the CDR as a bilevel problem. Their formulation has a lower level sub-problem for each pair of aircraft; optimal solutions of sub-problems correspond to time instants at which aircraft are closest. The constraints involving the lower levels ensure that the distance at this time instant is at least $d$ for each pair of aircraft. The proposed model serves for more than two dimensions, but here we present it in two to maintain our notation:

$$
\begin{array}{ccc}
\min _{q, t} & \sum_{i \in \mathcal{A}}\left(q_{i}-1\right)^{2} & \\
\text { s.t. } & \underline{q}_{i} \leq q_{i} \leq \bar{q}_{i} & \forall i \in \mathcal{A} \\
& \min _{t_{i j} \in[0, T]}\left[\left(\hat{x}_{i}-\hat{x}_{j}\right)+t_{i j}\left(q_{i} \hat{V}_{x i}-q_{j} \hat{V}_{x j}\right)\right]^{2} & \\
& +\left[\left(\hat{y}_{i}-\hat{y}_{j}\right)+t_{i j}\left(q_{i} \hat{V}_{y i}-q_{j} \hat{V}_{y j}\right)\right]^{2} \geq d^{2} & \forall i, j \in \mathcal{A} .
\end{array}
$$

\subsection{Heading angle changes}

The models presented in the previous section can be adapted to address CDR via $\mathrm{HAC}$, for instance, by modifying the decision variables. Indeed, to model HAC, Alonso-Ayuso et al. (2014) use a very similar formulation to that proposed in Pallottino et al. (2002) based on SC. The resulting formulation is a MINLP involving trigonometric functions of the variables, which are the heading angle variations. Another example is Cafieri \& Omheni (2017), where a two-step algorithm is proposed for the CDR. In each step, a MP formulation is solved: first, speed is regulated with model (29); then, if there are unsolved conflicts a HAC model is applied. The ideas behind the formulation considering $\mathrm{HAC}$ are pretty similar to that of $\mathrm{SC}$, and also 
involves trigonometric formulas:

$$
\begin{array}{ccc}
\min _{\omega, V, t^{m i n}, z} & \sum_{i \in \mathcal{A}} \omega_{i}^{2} & \\
\text { s.t. } & \underline{\omega}_{i} \leq \omega_{i} \leq \bar{\omega}_{i} & \forall i \in \mathcal{A} \\
& V_{i}=\left(\hat{v}_{i} \cos \left(\hat{\theta}_{i}+\omega_{i}\right), \hat{v}_{i} \sin \left(\hat{\theta}_{i}+\omega_{i}\right)\right) & \forall i \in \mathcal{A} \\
V_{i j}=V_{i}-V_{j} & \forall i, j \in \mathcal{A} \\
z_{i j}\left(\left\|V_{i j}\right\|^{2}\left(\hat{d}_{i j}^{2}-d^{2}\right)-\left\langle\hat{p}_{i j}, V_{i j}\right\rangle^{2}\right) \geq 0 & \forall i, j \in \mathcal{A} \\
t_{i j}^{m i n}=\frac{-\left\langle\hat{p}_{i j}, V_{i j}\right\rangle}{\left\|V_{i j}\right\|^{2}} & \forall i, j \in \mathcal{A} \\
t_{i j}^{m i n}\left(2 z_{i j}-1\right) \geq 0 & \forall i, j \in \mathcal{A} \\
z_{i j} \in\{0,1\} & \forall i, j \in \mathcal{A} .
\end{array}
$$

Similarly, Cerulli et al. (2020) also propose a bilevel programming formulation via HAC, which reads:

$$
\begin{array}{ccc}
\min _{\omega, t} & \sum_{i \in \mathcal{A}} \omega_{i}^{2} \\
\text { s.t. } & \underline{\omega}_{i} \leq \omega_{i} \leq \bar{\omega}_{i} & \\
& \min _{t_{i j} \in[0, T]}\left[\left(\hat{x}_{i}-\hat{x}_{j}\right)+t_{i j}\left(\hat{v}_{i} \cos \left(\hat{\theta}_{i}+\omega_{i}\right)-\hat{v}_{j} \cos \left(\hat{\theta}_{j}+\omega_{j}\right)\right)\right]^{2} & \\
& +\left[\left(\hat{y}_{i}-\hat{y}_{j}\right)+t_{i j}\left(\hat{v}_{i} \sin \left(\hat{\theta}_{i}+\omega_{i}\right)-\hat{v}_{j} \sin \left(\hat{\theta}_{j}+\omega_{j}\right)\right)\right]^{2} \geq d^{2} \quad \forall i, j \in \mathcal{A} .
\end{array}
$$

On the other hand, Pallottino et al. (2002) propose a completely different formulation for HAC. However, it is only valid when all the aircraft fly at the same speed. The formulation, which is not explicitly written in their paper, is based on equations (7), which are used for pairs of aircraft with crossing trajectories, and another set of separation constraints, which the authors propose for non-crossing trajectories in the direction of motion (which might become crossing trajectories due to heading angle changes). Here, for simplicity, we present a MILP based uniquely on (7), which is enough to model CDR since these conditions are equivalent to (3), as shown in Section 3.1. The authors distinguish three cases in order to model (7), namely (i) $\left(\theta_{i}+\theta_{j}+\pi\right) / 2 \in[-\pi, \pi]$, (ii) $\left(\theta_{i}+\theta_{j}+\pi\right) / 2>\pi$ and (ii) $\left(\theta_{i}+\theta_{j}+\pi\right) / 2<-\pi$. The decision variables are the heading angle deviations $\omega_{i}$ for each $i \in \mathcal{A}$, where $\theta_{i}=\hat{\theta}_{i}+\omega_{i}$. According to Pallottino et al., fourteen groups of linear constraints and six groups of binary variables are 
used to model (7). Here, we reconstruct the resulting formulation as follows:

$$
\begin{aligned}
& \min _{\omega, \nu, z} \quad \sum_{i \in \mathcal{A}} \nu_{i} \\
& \text { s.t. } \quad-\nu_{i} \leq \omega_{i} \leq \nu_{i} \quad \forall i \in \mathcal{A}(36 \mathrm{~b}) \\
& \underline{\omega}_{i} \leq \omega_{i} \leq \bar{\omega}_{i} \quad \forall i \in \mathcal{A}(36 \mathrm{c}) \\
& \left.\omega_{i}+\omega_{j} \leq \pi-\hat{\theta}_{i}-\hat{\theta}_{j}+M\left(1-z_{i j}^{1}\right) \quad \forall i, j \in \mathcal{A} 36 \mathrm{~d}\right) \\
& -\omega_{i}-\omega_{j} \leq-3 \pi+\hat{\theta}_{i}+\hat{\theta}_{j}+M\left(1-z_{i j}^{1}\right) \quad \forall i, j \in \mathcal{A}(36 \mathrm{e}) \\
& \omega_{i}+\omega_{j} \leq 2 \tan \vartheta_{i j}^{-}-\pi-\hat{\theta}_{i}-\hat{\theta}_{j}+M\left(1-z_{i j}^{1}\right) \quad \forall i, j \in \mathcal{A}(36 \mathrm{f}) \\
& \left.\omega_{i}+\omega_{j} \leq \pi-\hat{\theta}_{i}-\hat{\theta}_{j}+M\left(1-z_{i j}^{2}\right) \quad \forall i, j \in \mathcal{A} 36 \mathrm{~g}\right) \\
& \left.-\omega_{i}-\omega_{j} \leq-3 \pi+\hat{\theta}_{i}+\hat{\theta}_{j}+M\left(1-z_{i j}^{2}\right) \quad \forall i, j \in \mathcal{A} 36 \mathrm{~h}\right) \\
& -\omega_{i}-\omega_{j} \leq-2 \tan \vartheta_{i j}^{+}+\pi+\hat{\theta}_{i}+\hat{\theta}_{j}+M\left(1-z_{i j}^{2}\right) \quad \forall i, j \in \mathcal{A}(36 \mathrm{i}) \\
& -\omega_{i}-\omega_{j} \leq-\pi+\hat{\theta}_{i}+\hat{\theta}_{j}+M\left(1-z_{i j}^{3}\right) \quad \forall i, j \in \mathcal{A}(36 \mathrm{j}) \\
& \left.\omega_{i}+\omega_{j} \leq 2 \tan \vartheta_{i j}^{-}+\pi-\hat{\theta}_{i}-\hat{\theta}_{j}+M\left(1-z_{i j}^{3}\right) \quad \forall i, j \in \mathcal{A} 36 \mathrm{k}\right) \\
& -\omega_{i}-\omega_{j} \leq-\pi+\hat{\theta}_{i}+\hat{\theta}_{j}+M\left(1-z_{i j}^{4}\right) \quad \forall i, j \in \mathcal{A}(36 \mathrm{l}) \\
& -\omega_{i}-\omega_{j} \leq-2 \tan \vartheta_{i j}^{+}-\pi+\hat{\theta}_{i}+\hat{\theta}_{j}+M\left(1-z_{i j}^{4}\right) \quad \forall i, j \in(\mathrm{B} 6 \mathrm{~m}) \\
& \left.\omega_{i}+\omega_{j} \leq-3 \pi-\hat{\theta}_{i}-\hat{\theta}_{j}+M\left(1-z_{i j}^{5}\right) \quad \forall i, j \in \mathcal{A} 36 \mathrm{n}\right) \\
& \left.\omega_{i}+\omega_{j} \leq 2 \tan \vartheta_{i j}^{-}-3 \pi-\hat{\theta}_{i}-\hat{\theta}_{j}+M\left(1-z_{i j}^{5}\right) \quad \forall i, j \in \mathcal{A} 36 \mathrm{o}\right) \\
& \left.\omega_{i}+\omega_{j} \leq-3 \pi-\hat{\theta}_{i}-\hat{\theta}_{j}+M\left(1-z_{i j}^{6}\right) \quad \forall i, j \in \mathcal{A} 36 \mathrm{p}\right) \\
& \left.-\omega_{i}-\omega_{j} \leq-2 \tan \vartheta_{i j}^{+}+3 \pi+\hat{\theta}_{i}+\hat{\theta}_{j}+M\left(1-z_{i j}^{6}\right) \quad \forall i, j \in \mathcal{A} 36 \mathrm{q}\right) \\
& z_{i j}^{1}+z_{i j}^{2}+z_{i j}^{3}+z_{i j}^{4}+z_{i j}^{5}+z_{i j}^{6} \geq 1 \quad \forall i, j \in \mathcal{A}(36 \mathrm{r}) \\
& z_{i j}^{1}, z_{i j}^{2}, z_{i j}^{3}, z_{i j}^{4}, z_{i j}^{5}, z_{i j}^{6} \in\{0,1\} \quad \forall i, j \in \mathcal{A} .
\end{aligned}
$$

The objective function (36a) is to minimize de 1-norm of the vector of heading angle deviations. Indeed, due to $(36 \mathrm{~b}), \nu_{i}=\left|\omega_{i}\right|$ for all $i \in \mathcal{A}$ in any optimal solution. The group of constraints (36d)-(36i), ensure aircraft separation when $\left(\theta_{i}+\theta_{j}+\pi\right) / 2 \in[-\pi, \pi]$; the group $(36 \mathrm{j})-(36 \mathrm{~m})$ is active when $\left(\theta_{i}+\theta_{j}+\pi\right) / 2>\pi$, and impose condition (7) shifted in $\pi$ units; finally, 
(36n)-(36q) stand for (7) shifted in $-\pi$ units when $\left(\theta_{i}+\theta_{j}+\pi\right) / 2<-\pi$. Finally, (36r) ensure that at least one of the previous six groups of constraints is active.

\subsection{Speed and heading angle changes}

Frazzoli et al. (2001) write the CDR as a quadratically constrained quadratic program. Their decision variables represent the change made to nominal velocity vectors. That is, they are defined as $U_{i}$ such that $V_{i}=\hat{V}_{i}+U_{i}$ for all $i \in \mathcal{A}$. Consequently, their model implicitly allows both speed and heading angle changes. They consider given preferred deviations, $\hat{U}_{i}$. The proposed MP formulation reads:

$$
\begin{array}{ccc}
\min _{U, W} & \sum_{i \in \mathcal{A}}\left\|\hat{U}_{i}-U_{i}\right\|^{2} & \\
\text { s.t. } & \left\|\hat{V}_{i}+U_{i}\right\| \leq \bar{v} & \forall i \in \mathcal{A} \\
& \frac{\left\langle\left(\hat{V}_{i}+U_{i}\right), \hat{V}_{i}\right\rangle}{\left\|\hat{V}_{i}\right\|} \geq \underline{v} & \forall i \in \mathcal{A} \\
\left\langle\left(\hat{V}_{i j}+U_{i j}\right), \hat{p}_{i j}\right\rangle+W_{i j} \sqrt{\hat{d}_{i j}^{2}-d^{2}} \geq 0 & \forall i, j \in \mathcal{A} \\
\left\|\hat{V}_{i j}+U_{i j}\right\|^{2} \geq W_{i j}^{2} & \forall i, j \in \mathcal{A} \\
W_{i j} \geq 0 & \forall i, j \in \mathcal{A} .
\end{array}
$$

The objective function (37a) is to minimize the differences between the obtained deviations and the preferred ones. Constraints (37b) and (37c) stand for maneuvers bounds. On the one hand, (37b) ensure that the maximum speed is not exceeded. On the other hand, (37c) are a convex approximation to minimum speed constraints $\left\|\hat{V}_{i}+U_{i}\right\| \geq \underline{v}$. Indeed, observe that (37c) are equivalent to $\left\|\hat{V}_{i}+U_{i}\right\| \cos \left(\angle\left(\hat{V}_{i}+U_{i}, \hat{V}_{i}\right)\right) \geq \underline{v}$. Note that this also yields bounds on admissible heading angle changes. Finally, (37d) and (37e) are equivalent to separation constraints (12).

Omer \& Farges (2013) proposed a hybridization of nonlinear and mixed integer linear programming to tackle the SHAC version of the CDR. Their models feature uniformly accelerated aircraft motion, trajectory recovery, and obstacle avoidance. They first formulate the CDR as a Bolza problem, with continuous variables and infinitely many constraints in the domain of time. Then, time discretization is considered to build two alternative formulations, namely a nonlinear program with only continuous variables and a mixed integer linear program. The latter is a simplification of the 
former in which: (i) a linear approximation of convex quadratic constraints on maneuvers bounds is considered, and (ii) separation constraints (1) are approximated through disjunctive half-planes. The authors propose to use the optimal solution of the MILP as starting point when solving the NLP.

Omer (2015) develops a space-discretized model such as that in Vela et al. (2009b), Rey et al. (2014), which are typically based on speed regulation, that allows both speed and heading angle maneuvers. The author tries to model trajectories in a realistic way by considering vectors of velocity that are continuous with respect to time (instead of instantaneous maneuvers), and additional bounds on acceleration and yaw rate. To model the problem, a conflict graph is used: nodes are the origin and destination of aircraft plus the crossing points between trajectories, while edges link consecutive nodes through which an aircraft is planned to fly. The author derives different equations to account for the temporal and spatial shifts yielded by the different types of maneuvers. To linearize some of the model equations, a discrete set of possible heading changes is considered. The objective function takes into account both fuel consumption and time delay. Reproducing the proposed MP formulation would need from more notation and formulas than those already introduced, and is out of the scope of this survey. We refer the interested reader to the original paper for more details.

Alonso-Ayuso et al. (2016) present a MINLP in the same vein of (28), which is based on separation conditions (6). The authors use speed and heading angle variations as decision variables; trigonometric functions of the variables made their constraints highly nonlinear. Their model addresses the more general scenario in which altitude level reallocation are allowed, and it includes the SHAC variant as a particular case. As optimization goal, they consider a multi-objective criteria in which priorities between the three types of maneuvers are taken into account.

A different discrete optimization approach is presented by Lehouillier et al. (2017b), who also propose to use a graph as modelling tool. In this case, nodes stand for aircraft maneuvers, while edges connect those that are conflict-free. The authors identify the CDR with a variant of the minimumweight maximum-clique problem. In doing so, they also propose a way of computing maneuvers costs. To identify conflict-free maneuvers, they use a time discretization and evaluate separation condition (1) either on the extremes of the considered time intervals or on the time at which the aircraft are closest (which can be analytically obtained). One of the advantages of the proposed framework is that it is valid for any choice of available maneuvers. The model is generalized to handle uncertainties in Lehouillier et al. (2017a). The authors consider errors due to wind, imprecision on 
aircraft speeds, and delay in the execution of maneuvers.

Rey \& Hijazi (2017b) proposed a MINLP with the following variables:

$$
\begin{aligned}
\delta_{x i}:=q_{i} \cos \omega_{i} & \forall i \in \mathcal{A}, \\
\delta_{y i}:=q_{i} \sin \omega_{i} & \forall i \in \mathcal{A} .
\end{aligned}
$$

These variables allow to write the constraints of their model in complex number notation. However, the formulation we present here has been taken from their implementation available in GitHub (see Rey \& Hijazi 2017a). There, the following short-hands are used:

$$
a_{i j}=\hat{y}_{i j}^{2}-d^{2}, \quad b_{i j}=\hat{x}_{i j}^{2}-d^{2}, \quad c_{i j}=2 \hat{x}_{i j} \hat{y}_{i j} .
$$

Their formulation for CDR via SHAC is:

$$
\begin{aligned}
& \min _{\delta, V, z} \quad \sum_{i \in \mathcal{A}} \delta_{y i}^{2}+\left(1-\delta_{x i}\right)^{2} \\
& \text { s.t. } \quad V_{x i j}=\delta_{x i} \hat{v}_{i} \cos \hat{\theta}_{i}-\delta_{y i} \hat{v}_{i} \sin \hat{\theta}_{i}-\delta_{x j} \hat{v}_{j} \cos \hat{\theta}_{j}+\delta_{y j} \hat{v}_{j} \sin \hat{\theta}_{j} \quad \forall i, j \in \mathcal{A} \\
& V_{y i j}=\delta_{y i} \hat{v}_{i} \cos \hat{\theta}_{i}+\delta_{x i} \hat{v}_{i} \sin \hat{\theta}_{i}-\delta_{y j} \hat{v}_{j} \cos \hat{\theta}_{j}-\delta_{x j} \hat{v}_{j} \sin \hat{\theta}_{j} \quad \forall i, j \in \mathcal{A} \\
& -z_{i j} M_{i j} \leq V_{y i j} \hat{x}_{i j}-V_{x i j} \hat{y}_{i j} \leq\left(1-z_{i j}\right) M_{i j} \quad \forall i, j \in \mathcal{A} \\
& 2 a_{i j} V_{x i j}-V_{y i j}\left(c_{i j}-\sqrt{c_{i j}^{2}-4 a_{i j} b_{i j}}\right) \leq\left(1-z_{i j}\right) M_{i j} \quad \forall i, j \in \mathcal{A}: \hat{x}_{i j} \geq 0, \hat{y}_{i j} \text { (4⿴囗⿱一一) } \\
& -2 b_{i j} V_{y i j}+V_{x i j}\left(c_{i j}-\sqrt{c_{i j}^{2}-4 a_{i j} b_{i j}}\right) \leq z_{i j} M_{i j} \quad \forall i, j \in \mathcal{A}: \hat{x}_{i j} \geq 0, \hat{y}_{i j} \text { (4丹f) } \\
& -2 a_{i j} V_{x i j}+V_{y i j}\left(c_{i j}-\sqrt{c_{i j}^{2}-4 a_{i j} b_{i j}}\right) \leq\left(1-z_{i j}\right) M_{i j} \quad \forall i, j \in \mathcal{A}: \hat{x}_{i j}<0, \hat{y}_{i j}(\underline{\theta} \mathrm{g} 0 \\
& 2 b_{i j} V_{y i j}-V_{x i j}\left(c_{i j}-\sqrt{c_{i j}^{2}-4 a_{i j} b_{i j}}\right) \leq z_{i j} M_{i j} \quad \forall i, j \in \mathcal{A}: \hat{x}_{i j}<0, \hat{y}_{i j}(4 \underline{\theta} \underline{\mathrm{h}} \text { ) } \\
& 2 b_{i j} V_{y i j}-V_{x i j}\left(c_{i j}-\sqrt{c_{i j}^{2}-4 a_{i j} b_{i j}}\right) \leq\left(1-z_{i j}\right) M_{i j} \quad \forall i, j \in \mathcal{A}: \hat{x}_{i j} \geq 0, \hat{y}_{i \text { ( }} \text { (些i) } \\
& 2 a_{i j} V_{x i j}-V_{y i j}\left(c_{i j}-\sqrt{c_{i j}^{2}-4 a_{i j} b_{i j}}\right) \leq z_{i j} M_{i j} \quad \forall i, j \in \mathcal{A}: \hat{x}_{i j} \geq 0, \hat{y}_{i j}(\underline{40 j \mathrm{j})} \\
& \left.-2 b_{i j} V_{y i j}+V_{x i j}\left(c_{i j}-\sqrt{c_{i j}^{2}-4 a_{i j} b_{i j}}\right) \leq\left(1-z_{i j}\right) M_{i j} \quad \forall i, j \in \mathcal{A}: \hat{x}_{i j}<0, \hat{y}_{i j} 4 \notin k \mathrm{k}\right) \\
& -2 a_{i j} V_{x i j}+V_{y i j}\left(c_{i j}-\sqrt{c_{i j}^{2}-4 a_{i j} b_{i j}}\right) \leq z_{i j} M_{i j} \quad \forall i, j \in \mathcal{A}: \hat{x}_{i j}<0, \hat{y}_{i j}(4010 \\
& \underline{v}^{2} \leq \delta_{x i}^{2}+\delta_{y i}^{2} \leq \bar{v}^{2} \quad \forall i \in \mathcal{A} \\
& \delta_{x i} \tan \underline{\omega} \leq \delta_{y i} \leq \delta_{x i} \tan \bar{\omega} \quad \forall i \in \mathcal{A} \\
& z_{i j} \in\{0,1\} \quad \forall i, j \in \mathcal{A}
\end{aligned}
$$

The authors propose to minimize (40a) since each term in the summation is equal to $q_{i}^{2}-2 q_{i} \cos \omega_{i}+1$, which is minimal when $q_{i}=1$ and $\omega_{i}=$ 
0. Constraints (40b) and (40c) state the relation between the vector of relative velocity and variables $\delta$. On the other hand, (40d)-(401) define the different regions in the space $\left(V_{x i j}, V_{y i j}\right)$ where aircraft separation is maintain, which were described at the end of Section 3.2. Finally, (40m) impose speed regulation bounds, while (40n) establish the limits on heading angle variations. Note that the inequalities of the left-hand side of $(40 \mathrm{~m})$ define non-convex constraints and that the formulation is linear except for the objective and the bounds on the speed.

Finally, Dias et al. (2020) adopt and extend (40) to derive non-convex MIP formulations for the SHAC and to propose exact optimization approaches based on tightened convex relaxations. The authors address an extended problem where flight level changes are considered as well.

\section{Optimal solution computation}

As we have seen, CDR is a complex task that, even after simplifications, requires from nonlinear equations and binary variables to be modelled as a mathematical program. Here, we try to give a general picture on how far these models have gone when solving CDR instances. We do not aim at comparison between different formulations or solving techniques. On the one hand, few benchmarking instances have been shared across studies; on the other hand, computational resources and configurations always vary. Instead of that, we discuss on which types of CDR benchmarks have appeared on existing literature, and we provide an idea of which are the limits of the different MP approaches.

There are a few sets of instances that have been commonly used in previous works. They correspond either to predefined scenarios or just to randomly generated configurations. Existing benchmarks of the CDR problem in the literature include:

- Circle instances. Aircraft initial positions are arranged on a circumference of a given radius, and they fly towards the center following the rectilinear trajectory given by the corresponding diameter. The circle radius is usually $100 \mathrm{NM}$ or $200 \mathrm{NM}$ and nominal speed is typically considered to be $400 \mathrm{NM} / \mathrm{h}$ for all aircraft. This scenario is widely known as the circle problem, even though it has also been called roundabout, e.g. in Omer \& Farges (2013) and Omer (2015), and symmetric encounter pattern, see Frazzoli et al. (2001). Aircraft are usually distributed on the circumference in a uniform way, as illustrated by Figure 7a. This is the case of the instances considered by Rey et al. 


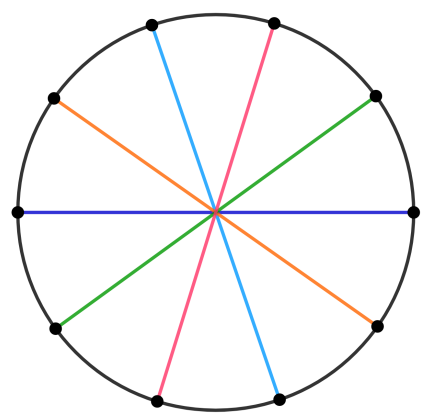

(a) Circle with 10 aircraft

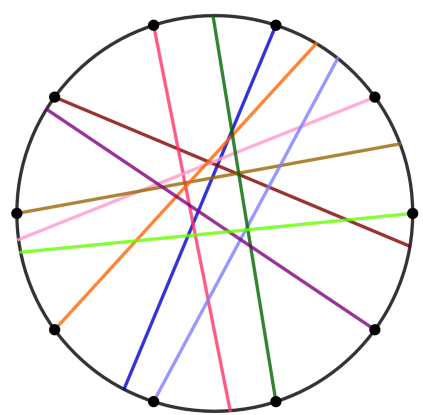

(c) Random circle with 10 aircraft

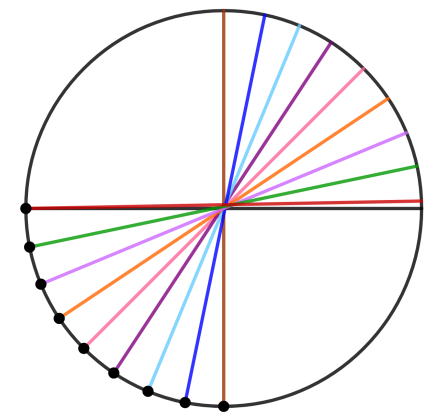

(b) Circle, one quarter variant

Figure 7: Circle instances 


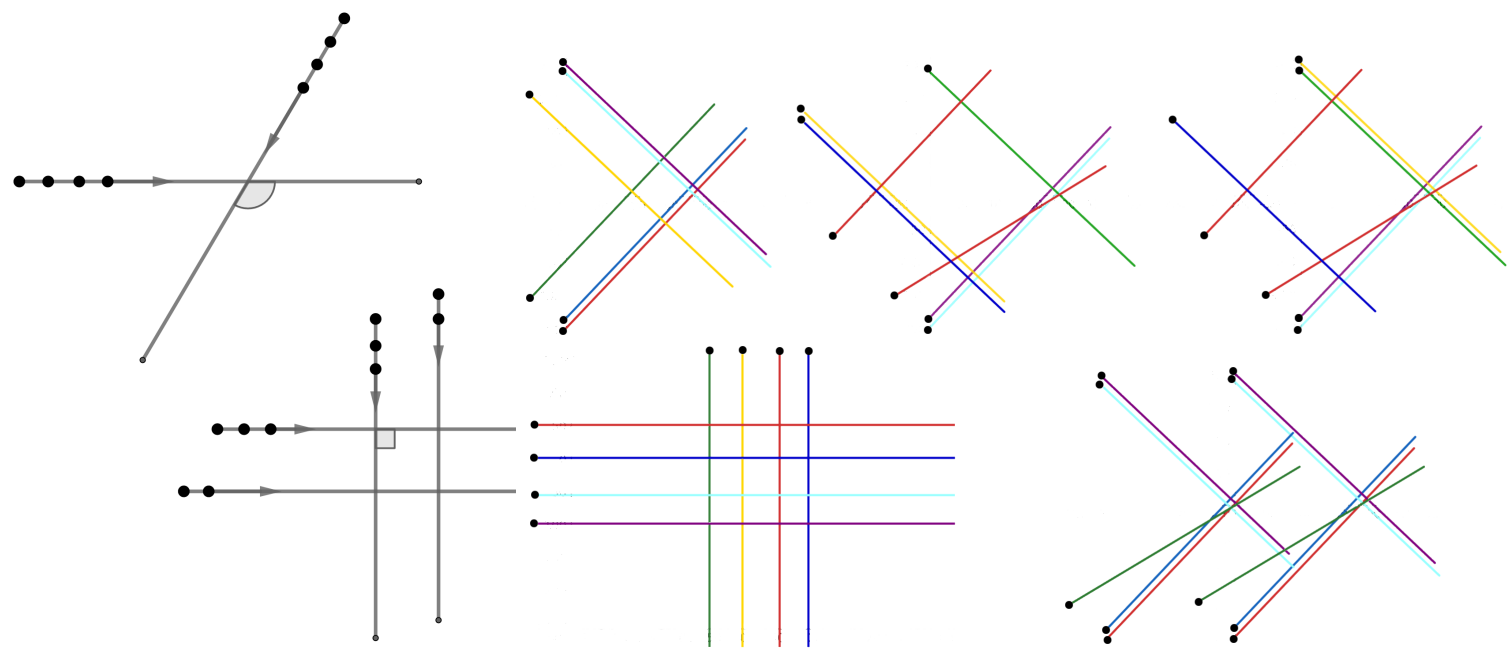

(a) Scenario types used by Omer (b) Five scenarios used by Cafieri \& Durand (2015) (2014)

Figure 8: Grid/Rhomboidal instances

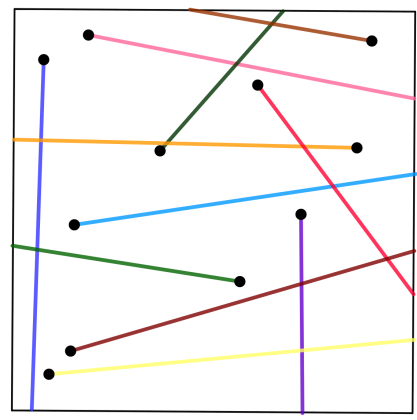

Figure 9: Random instance 
(2014, 2016), Cafieri \& Rey (2017), Rey \& Hijazi (2017b), Dias et al. (2020). In other works, such as Cafieri \& Durand (2014), Rey et al. (2016), Cafieri \& D'Ambrosio (2018), Cerulli et al. (2020), aircraft are distributed on a quarter of the circumference and their trajectories can be slightly deviated (a random quantity between $-5^{\circ}$ and $5^{\circ}$ ) from the center, see Figure 7b. Note that in the latter configuration frontal conflicts are neglected.

- Random circle instances. The previous family gathers very unrealistic scenarios. In order to obtain more realistic configurations, they have been modified by considering a random deviation between $-30^{\circ}$ and $30^{\circ}$ from diametrical trajectories. An example of a random circle instance with 10 aircraft is given by Figure 7c. This kind of instance has been used in works such as Rey et al. (2014, 2016), Cafieri \& Rey (2017), Rey \& Hijazi (2017b), Dias et al. (2020). There are circle and random circle instances publicly available at the GitHub repository Rey \& Hijazi (2017a).

- Grid/Rhomboidal instances. In these scenarios, aircraft fly on rectilinear trajectories crossing at different points of the space. When the trajectories cross at right angles, the scenario is called grid. Sometimes, several aircraft are flying on a trail configuration, following each other on a same stream. This is the case for instance in Frazzoli et al. (2001), Omer \& Farges (2013), Omer (2015), Dias et al. (2020), which include aircraft displayed on two or four streams in their studies (see Figure 8a). Other times, each aircraft follows an independent trajectory, like in the five scenarios depicted by Figure 8b. These were introduced as opposed to circle instances in Cafieri \& Durand (2014), and then used in Cafieri \& D'Ambrosio (2018), Cerulli et al. (2020). The figure shows rectilinear trajectories crossing at different points and corresponding to different aircraft, ranging between 6 and 10 .

- Random trajectory instances. In this case both initial positions and aircraft velocity vectors are randomly generated, see Frazzoli et al. (2001), Alonso-Ayuso et al. (2016), Cafieri \& Rey (2017). These benchmarks are designed to represent random configurations where some aircraft may be diverging and/or initially violating the $5 \mathrm{NM}$ separation standard. Figure 9 illustrates a random trajectory instance. The airspace is frequently considered to be a square, with $100 \mathrm{NM}$ each side and aircraft flying at same speed, $400 \mathrm{NM} / \mathrm{h}$. 
In order to give an idea of the state-of-the-art models performances on the above-mentioned instances, we focus on most recent approaches. Our exposition is organized based on the variant addressed by the models, namely SC, HAC or SHAC.

Cafieri \& Durand (2014) propose to solve their formulation for the SC problem with the global solver CoUENNE or, alternatively, with a tailored heuristic algorithm. Their heuristic decomposes the SC problem into smaller sub-problems. The exact solutions of the sub-problems are then combined to form a globally feasible but possibly sub-optimal solution of the original problem. They could solve circle instances of the type depicted on Figure $7 \mathrm{~b}$ with up to 10 aircraft in 759.40 seconds; and the grid and rhomboidal benchmarks depicted on Figure 8b in 3731.43 seconds at most. A different heuristic for SC namely to solve the formulation (30), is proposed in Cafieri \& D'Ambrosio (2018). In this case, the authors propose a feasibility pump heuristic that builds two sequences of solutions: ones that are feasible with respect to nonlinear constraints, and other satisfying the integrality conditions. The algorithm iterates until the two sequences converge to a feasible solution of (30). The authors used the same benchmarks as Cafieri \& Durand to test their heuristic. CPU time is less than 640 seconds for circle instances and 200 seconds for non-circle ones.

Instead of minimizing deviation, there are other approaches based on SC that aim at minimizing conflicts. This is the case of Rey et al. (2014) and Rey et al. (2016). The first test their equity-oriented conflict resolution model on circle and random circle instances having up to 11 and 30 aircraft respectively. They use CPLEx to solve the MP formulations at the three stages of their model. Solving one stage took on average 35 seconds for the biggest circle instance and 25 seconds for the random circle with 30 aircraft. On the other hand, Rey et al. (2016) propose two formulations to maximize the conflicts solved and minimize total conflicts duration, which were solved using Cplex. Tests on circle and random circle instances show that the performance of both formulations is comparable, solving the circle instance of size 10 in less than 250.50 seconds and a random circle with 30 aircraft in less than 42.9 .

In the same vein, Cafieri \& Rey (2017) propose several MINLP formulations to reduce the number of conflicts via SC. They consider two models, one aiming at maximizing the number of conflicts solved and another to identify the largest conflict-free set of aircraft. For each of them, they present two formulations, which differ in whether diverging trajectories are considered or not. Random trajectory instances are used to test the former, while random circle scenarios are considered for the latter since they do not 
include diverging trajectories. When the objective is to maximize the conflicts solved, they solve 7 out of 20 random circle instances of size 10 within a time limit of 300 seconds, and 2 out of 20 when the size is incremented to 20 aircraft. For random trajectory instances they use formulation (29), which solves the 20 instances of 10 aircraft tested in less than 5 seconds, and 9 out of 20 instances of size 20 within the time limit. In the case of the model that maximizes the size of the conflict-free set, they solved 5 out of 20 random circle instances of size 10 and 3 out of 20 with 20 aircraft, within the time limit. In this case the authors also propose a heuristic, which finds equally good or better solutions than Couenne, the solver chosen to solve the MP formulations. In the case of random trajectory instances, they can solve the 20 instances of size 10 tested in less than 33 seconds, while when size is increased to 20 aircraft they can only solve 2 out of 20 instances within the time limit. For these instances, the heuristic does not improve solution quality in general. Conversely, for 10 aircraft the optimal is found for 11 instances and for 20 aircraft the heuristic usually finds worse solutions, and only represents an improvement in three cases, for which the MP cannot find a feasible solution.

Cafieri \& Omheni (2017) propose a model to address both SC and HAC. Their procedure is made of two steps: first formulation (29) is used as a preprocessing to adjust aircraft speeds; then, the remaining conflicts are solved via HAC with formulation (34) and the speeds resulting from the previous step. Both MP formulations are solved with Couenne. Cafieri \& Omheni test their model also on circle instances. They report CPU times of 2819.51 seconds at most for the circle problem with 6 aircraft and 2561.98 seconds at most for random circle instances with 8 aircraft.

Omer (2015) used GuroBI to solved its space-discretized MILP, which models the CDR problem with SHAC. He solved circle scenarios with 6 aircraft in 124.4 seconds on average; grid configurations with 12 aircraft in 148.4 seconds on average; and rhomboidal scenarios with two crossing streams and 12 aircraft (see Figure 8a) in 142.2 seconds on average.

Alonso-Ayuso et al. (2016) propose three variants of a conflict resolution model based on SHAC. In order to prioritize the different maneuvers, each variant uses a different criterion for multi-objective optimization, namely lexicographic, compromise, and a mixture of minimizing the largest maneuver and the compromise criterion. The models were solved using Minotaur and tested on circle and random instances. For circle instances with up to 7 aircraft, largest CPU times range from 452.63 to 2200.77 seconds depending on the multiobjective criteria used (pre-calculation of so-called ideal values included). Similarly, for random trajectory instances up to 20 aircraft, worst 
CPU times range from 27.69 to 35.48 seconds.

Rey \& Hijazi (2017b) propose an algorithm that solves different relaxations of their complex number formulation (40) to obtain lower bounds and fix binary variables in (40) to obtain upper bounds. The algorithm is tested on circle instances with up to 20 aircraft and random circle ones having up to 40 aircraft. Circle instances with 4 to 10 aircraft are solved to global optimality within 73.36 seconds. In particular, instances with up to 7 aircraft are solved in less than a second. On the other hand, circle instances with 11 to 17 aircraft are solved to local optimality within the time limit of 300 seconds whereas feasible solutions for those of size 18, 19, and 20 needed 300 seconds more. To evaluate the performance of their approach on random circle instances, the authors generate 100 instances for each scenario size, i.e. 10, 20, 30, and 40 aircraft. All instances of size 10 and 20 are solved to global optimality in less than a second. For 30-aircraft instances, 83 are solved to global optimality, 71 in 4.35 seconds on average and 12 in 35.58 seconds on average. The remaining 17 instances are solved to local optimality. In the case of 40 aircraft, only 17 instances can be solved to optimality. The CPU time needed increases to 99.05 seconds on average for 16 of them. Local optimal solutions are found for 75 instances in 261.6 seconds on average, while no solution is found for the remaining 8 .

In summary, current approaches limits are around 20 aircraft for circle instances and 40 for random circle, whereas it is not clear for non-circle ones. Regarding circle instances, optimal solutions are known up to 10 aircraft, while instances from 11 to 20 aircraft remain open and only local optima are known. In the case of random circle, 100 instances of each size 10 and 20 are solved to optimality. The scenario becomes more challenging with the increase of aircraft. With 30, most of the instances can be solved in less than one minute, whereas with 40 we need more than 4 minutes to obtain a local solution in most cases. Finally, non-circle instances have not been so extensively used for testing. We know that random trajectory instances of 20 aircraft can be solved in less than one minute. On the other hand, specific grid and rhomboidal configurations of Figure 8 with up to 12 aircraft have been solved in 3.3 minutes at most.

\section{Future research directions}

Despite the fact that CDR optimization methods emerged to answer a need from real application, many of the key features for obtaining operationallyuseful solutions have not been still addressed. The methods described herein rely on simplifying assumptions that include co-planar motion, rectilinear 
trajectories, and perfect information. This limits their interest to the academic context rather than real ATC, which requires more flexible assumptions.

One of the features that should be improved is aircraft motion. Rectilinear trajectory assumption might hold often at cruising level, but also excludes other particular scenarios. Something similar happens with coplanarity: most challenging CDR often involve aircraft converging in both the vertical and horizontal dimensions. In addition, aircraft position has been treated as perfect information, while it is actually uncertain. Future developments should allow for curving and accelerating trajectories under uncertainty due to track falloff or sensor errors. Also linked to uncertainty, weather conditions should not be excluded from models. Indeed, these represent external conditions such as wind that affect aircraft motion. Considering such conditions sums an extra degree of complexity to models, which have to deal with a random component and provide robust solutions.

Regarding maneuvering, oversimplifications are also a significant shortcoming of current studies. First, maneuvers are frequently assumed to be performed at the beginning of the time horizon. This does not answer a real operational need and, moreover, it constitutes an important limitation of the feasible set of solutions. Modelling of trajectory recovery is another aspect that is still unsatisfying. Trajectory recovery responds to the operational concept of $4 \mathrm{D}$ contract, according to which trajectories must satisfy certain time and space requirements on a sequence of $4 \mathrm{D}$ points. After conflicts are avoided, aircraft must return to their original trajectories as soon as possible. Current models either rely on time discretization to address recovery at the end of the time horizon, or add penalizing terms in the objective for space and time shifts with respect to the nominal plan. However, time-discretized models require a granularity that is prohibitive in terms of performance, while penalizing terms do not effectively address the problem. Recovery is indeed not trivial since trajectories must be tracked for potential conflicts during this phase as well. At the same time, recovery implies the introduction of "breaking points" in the trajectories, which are usually assumed to be straight as was already discussed. This evidences the flaws of current assumptions, which are too tight to handle situations that constantly occur in real world.

Finally, automation of traffic control in emerging domains such as urban air mobility and autonomous vehicles is certainly a promising future research direction. In these envisioned air transportation concepts, innovative aircraft could safely and efficiently transport passengers and goods within and around dense urban areas. There is a consensus to deem scaled operations 
issued by automated traffic management systems backed by human-in-theloop supervision. Integration of urban air mobility operations in existing ATM systems is one of the hot topic of today's aviation. Conflict detection and resolution must consider here additional elements, such as obstacle avoidance, specific vehicle profiles, vertical climbing, noise impact, stricter time constraints, or aircraft power limitations.

\section{Concluding remarks}

This review evidences that Mathematical Programming has a lot to say in the development of decision support tools for ATM, and, in particular, for aircraft deconfliction. However, after several decades of effort, current approaches still suffer form important limitations when it comes to their real application.

One of the contributions of this analysis is the derivation of the aircraft pairwise separation conditions, for which we show the links and equivalency across six different equations. These conditions, which most natural modelling corresponds to non-convex quadratic mathematical constraints, are key for CDR formulations. Their direct application gives nonlinear non-convex models, while alternatives include simplifications that affect model reliability and/or disjunctive mathematical constraints. The modelling choice for aircraft separation conditions makes a significant difference between approaches. Thus, showing the mathematical relation among the alternative separation conditions in the literature can bring new light into the problem. On the other hand, we revisit CDR mathematical formulations across the last few decades, providing a cohesive discussion on the different approaches. A taxonomy has been proposed to compare them, which includes the type of separation condition as one of the classifying features. The analysis made on several MP approaches illustrates the variety of modelling choices and how they affect the resulting formulations. We hope it can inspire new proposals from the field in near future. Regarding solution computation, state-of-the-art approaches are able to solve hard symmetric instances in a few seconds, although the number of aircraft is still limited to several dozens.

Future approaches, other than meeting computational requirements due to the online nature of the problem, would need to consider a larger set of features than those of the models discussed here. These include, among others, the ability to handle uncertainty, accurate modelling of objectives such as energy consumption, robustness of the solution against failure and integration with weather conditions. On the other hand, a larger variety of 
reference benchmark sets is needed, which includes synthetic and realistic instances, in order to validate and compare approaches.

\section{Acknowledgments}

This publication was supported by the Chair "Integrated Urban Mobility", backed by L'X - École Polytechnique and La Fondation de l'École Polytechnique and sponsored by Uber. We wish to acknowledge the referees of this work, whose insightful comments and suggestions have significantly contributed to improve the paper.

Alliot, J., Bosc, J., Durand, N., \& Maugis, L. (1997). Cats: A complete air traffic simulator. In 16th DASC. AIAA/IEEE Digital Avionics Systems Conference. Reflections to the Future (pp. 708-714). Irvine, CA, USA volume 8.2-30. URL: https://doi.org/10.1109/DASC.1997.637271.

Alonso-Ayuso, A., Escudero, L., \& Martín-Campo, F. (2010). Collision avoidance in air traffic management: A mixed-integer linear optimization approach. IEEE Transactions on Intelligent Transportation Systems, 12, 47-57. URL: https: //doi.org/10.1109/TITS.2010.2061971.

Alonso-Ayuso, A., Escudero, L., \& Martín-Campo, F. (2014). Exact and approximate solving of the aircraft collision resolution problem via turn changes. Transportation Science, 50, 263-274. URL: https://doi.org/10.1287/ trsc. 2014.0557.

Alonso-Ayuso, A., Escudero, L., \& Martín-Campo, F. (2016). An exact multiobjective mixed integer nonlinear optimization approach for aircraft conflict resolution. Top, 24, 381-408. URL: https://doi.org/10.1007/ s11750-015-0402-z.

Bareiss, D., \& den Berg, J. V. (2013). Reciprocal collision avoidance for robots with linear dynamics using lqr-obstacles. In IEEE International Conference on Robotics and Automation (pp. 3847-3853). URL: https://doi.org/10. 1109/ICRA. 2013.6631118.

Barnhart, C., Belobaba, P., \& Odoni, A. (2003). Applications of operations research in the air transport industry. Transportation Science, 4, 368-391. URL: https://doi.org/10.1287/trsc.37.4.368.23276.

Bilimoria, K. (2000). A geometric optimization approach to aircraft conflict resolution. In 18th Applied aerodynamics conference (p. 4265). URL: https: //doi.org/10.2514/6.2000-4265.

Brochard, M. (2005). ERASMUS: En Route Air Traffic Soft Management Ultimate System. Technical Report Eurocontrol Experimental Center.

Cafieri, S., \& D'Ambrosio, C. (2018). Feasibility pump for aircraft deconfliction with speed regulation. Journal of Global Optimization, 71, 501-515. URL: https://doi.org/10.1007/s10898-017-0560-7. 
Cafieri, S., \& Durand, N. (2014). Aircraft deconfliction with speed regulation: new models from mixed-integer optimization. Journal of Global Optimization, 58, 613-629. URL: https://doi.org/10.1007/s10898-013-0070-1.

Cafieri, S., \& Omheni, R. (2017). Mixed-integer nonlinear programming for aircraft conflict avoidance by sequentially applying velocity and heading angle changes. European journal of operational research, 260, 283-290. URL: https://doi.org/10.1016/j.ejor.2016.12.010.

Cafieri, S., \& Rey, D. (2017). Maximizing the number of conflict-free aircraft using mixed-integer nonlinear programming. Computers and Operations Research, 80, 147-158. URL: https://doi.org/10.1016/j.cor.2016.12.002.

Carlier, J., Nace, D., Duong, V., \& Nguyen, H. (2003). Using disjunctive scheduling for a new sequencing method in multiple-conflicts solving. In the 2003 IEEE International Conference on Intelligent Transportation Systems (pp. 708-714). volume 1. URL: https://doi.org/10.1109/ITSC.2003.1252043.

Cerulli, M., D'Ambrosio, C., Liberti, L., \& Pelegrín, M. (2020). Detecting and solving aircraft conflicts using bilevel programming. URL: https://hal. archives-ouvertes.fr/hal-02869699v3.

Courchelle, V., Soler, M., González-Arribas, D., \& Delahaye, D. (2019). A simulated annealing approach to 3D strategic aircraft deconfliction based on en-route speed changes under wind and temperature uncertainties. Transportation Research Part C: Emerging technologies, 103, 194-210. URL: https://doi. org/10.1016/j.trc.2019.03.024.

Dias, F., Hijazi, H., \& Rey, D. (2020). Disjunctive linear separation conditions and mixed-integer formulations for aircraft conflict resolution. URL: https: //arxiv.org/abs/1911.12997v3.

European Commission, \& EUROCONTROL (2009). Sesar consortium: The European ATM master plan. Technical Report 1.

Fairbrother, J., Zografos, K., \& Glazebrook, K. (2020). A slot-scheduling mechanism at congested airports that incorporates efficiency, fairness, and airline preferences. Transportation Science, 54, 115-138. URL: https://doi.org/ 10.1287/trsc.2019.0926.

Frazzoli, E., Mao, Z.-H., Oh, J.-H., \& Feron, E. (2001). Resolution of conflicts involving many aircraft via semidefinite programming. Journal of Guidance, Control and Dynamic, 24, 79-86. URL: https://doi.org/10.2514/2.4678.

Hansman, J. (2012). Impact of NextGen integration on improving efficiency and safety of operations. In TRB: the 91st Annual Meeting of the Transportation Research Board. Washington D.C, USA.

Huang, S., Teo, R., \& Tan, K. (2019). Collision avoidance of multi unmanned aerial vehicles: A review. Annual Reviews in Control, . URL: https://doi.org/ 10.1016/j. arcontrol.2019.10.001.

ICAO (1996). Rules of the air and air traffic services. Technical Report International Civil Aviation Organization. 
Irvine, R. (2001). A simplified approach to conflict probability estimation. In 20th DASC. 20th Digital Avionics Systems Conference (pp. 7F5-1). IEEE volume 2. URL: https://doi.org/10.1109/DASC.2001.964214.

Irvine, R. (2002). A geometrical approach to conflict probability estimation. Air Traffic Control Quarterly, 10, 85-113. URL: https://doi.org/10.2514/ atcq.10.2.85.

Kuchar, J., \& Yang, L. (2000). A review of conflict detection and resolution modeling methods. IEEE Transactions on intelligent transportation systems, 1, 179-189. URL: https://doi.org/10.1109/6979.898217.

Lehouillier, T., Nasri, M., Omer, J., Soumis, F., \& Desaulniers, G. (2017a). Solving the air conflict resolution problem under uncertainty using an iterative biobjective mixed integer programming approach. Transportation Science, 51, 1242-1258. URL: https://doi.org/10.1287/trsc.2016.0714.

Lehouillier, T., Omer, J., Soumis, F., \& Desaulniers, G. (2017b). Two decomposition algorithms for solving a minimum weight maximum clique model for the air conflict resolution problem. European Journal of Operational Research, 256, 696-712. URL: https://doi.org/10.1016/j.ejor.2016.07.008.

Liang, Z., Chaovalitwongse, W., \& Elsayed, E. (2014). Sequence assignment model for the flight conflict resolution problem. Transportation Science, 48, 334-350. URL: http://dx.doi.org/10.1287/trsc.2013.0480.

Mori, R. (2017). Fuel saving by gradual climb procedure. 7th SESAR Innovation Days.

Mourad, A., Puchinger, J., \& Chu, C. (2019). A survey of models and algorithms for optimizing shared mobility. Transportation Research Part B: Methodological, 123, 323-346. URL: https://doi.org/10.1016/j.trb.2019.02.003.

Omer, J. (2015). A space-discretized mixed-integer linear model for air-conflict resolution with speed and heading maneuvers. Computers and Operations Research, 58, 75-86. URL: https://doi.org/10.1016/j.cor.2014.12.012.

Omer, J., \& Farges, J. (2013). Hybridization of Nonlinear and Mixed-Integer Linear Programming for aircraft separation with trajectory recovery. IEEE Transactions on Intelligent Transportation Systems, 14, 1218-1230. URL: https://doi.org/10.1109/TITS.2013.2257758.

Pallottino, L., Feron, E., \& Bicchi, A. (2002). Conflict resolution problems for air traffic management systems solved with mixed integer programming. IEEE transactions on intelligent transportation systems, 3, 3-11. URL: https:// doi.org/10.1109/6979.994791.

Rey, D. (2020). Personal communication.

Rey, D., \& Hijazi, H. (2017a). AMPL models and data files from the CDC 2017 paper complex number formulation and convex relaxations for aircraft conflict resolution. URL: https://github.com/ReyHijazi/Conflict_Resolution.

Rey, D., \& Hijazi, H. (2017b). Complex number formulation and convex relaxations for aircraft conflict resolution. IEEE 56th Annual Conference on Decision and Control, (pp. 88-93). URL: https://doi.org/10.1109/CDC.2017.8263648. 
Rey, D., Rapine, C., Dixit, V., \& Waller, S. (2014). Equity-oriented aircraft collision avoidance model. IEEE Transactions on Intelligent Transportation Systems, 16, 172-183. URL: https://doi.org/10.1109/TITS.2014.2329012.

Rey, D., Rapine, C., Fondacci, R., \& Faouzi, N. E. (2012). Minimization of potential air conflicts through speed regulation. Transportation research record, 2300, 59-67. URL: https://doi.org/10.3141/2300-07.

Rey, D., Rapine, C., Fondacci, R., \& Faouzi, N. E. (2016). Subliminal speed control in air traffic management: Optimization and simulation. Transportation Science, 50, 240-262. URL: https://doi.org/10.1287/trsc.2015.0602.

Richards, A., \& How, J. (2002). Aircraft trajectory planning with collision avoidance using mixed integer linear programming. 2002 American Control Conference, 3, 1936-1941. URL: https://doi.org/10.1109/ACC.2002.1023918.

Rios, J. (2018). Strategic deconfliction: System requirements.

Samà, M., D'Ariano, A., D'Ariano, P., \& Pacciarelli, D. (2017). Scheduling models for optimal aircraft traffic control at busy airports: tardiness, priorities, equity and violations considerations. Omega, 67, 81-98. URL: https://doi.org/ 10.1016/j . omega. 2016.04.003.

Tarnopolskaya, T., \& Fulton, N. (2009). Optimal cooperative collision avoidance strategy for coplanar encounter: Merz's solution revisited. Journal of optimization theory and applications, 140, 355-375. URL: https://doi .org/10. 1007/s10957-008-9452-9.

Vela, A., Salaun, E., Solak, S., Feron, E., Singhose, W., \& Clarke, J. (2009a). A two-stage stochastic optimization model for air traffic conflict resolution under wind uncertainty. In IEEE/AIAA 28th Digital Avionics Systems Conference (pp. 2-E). URL: https://doi.org/10.1109/DASC.2009.5347531.

Vela, A., Solak, S., Clarke, J., Singhose, W., Barnes, E., \& Johnson, E. (2010). Near real-time fuel-optimal en route conflict resolution. IEEE Transactions on Intelligent Transportation Systems, 11, 826-837. URL: https://doi .org/ 10.1109/TITS. 2010.2051028.

Vela, A., Solak, S., Singhose, W., \& Clarke, J. (2009b). A mixed integer program for flight-level assignment and speed control for conflict resolution. In the $48 \mathrm{~h}$ IEEE Conference on Decision and Control (CDC) (pp. 5219-5226). URL: https://doi.org/10.1109/CDC.2009.5400520.

Wu, C., \& Caves, R. (2002). Research review of air traffic management. Transport Reviews, 22, 115-132. URL: https://doi.org/10.1080/ 01441640110074773.

Zohrevandi, E., Westin, C., Lundberg, J., \& Ynnerman, A. (2020). Design of a real time visual analytics support tool for conflict detection and resolution in air traffic control. In Joint conferences of Eurographics and Eurovis. 